\title{
Charged particle dynamics in multiple colliding electromagnetic waves. Survey of random walk, Lévy flights, limit circles, attractors and structurally determinate patterns
}

\author{
S. V. Bulanov ${ }^{1} \dagger$, T. Zh. Esirkepov ${ }^{1}$, J. K. Koga ${ }^{1}$, S. S. Bulanov ${ }^{2}$, Z. Gong ${ }^{3}$, \\ X. Q. Yan $^{3,4}$ and M. Kando \\ ${ }^{1}$ Department of Advanced Photon Research, Kansai Photon Research Institute, \\ National Institutes for Quantum and Radiological Science and Technology, 8-1-7 Umemidai, \\ Kizugawa-shi, Kyoto 619-0215, Japan \\ ${ }^{2}$ Lawrence Berkeley National Laboratory, Berkeley, CA 94720, USA \\ ${ }^{3}$ State Key Laboratory of Nuclear Physics and Technology, and Key Laboratory of HEDP of the Ministry \\ of Education, CAPT, Peking University, Beijing 100871, China \\ ${ }^{4}$ Collaborative Innovation Center of Extreme Optics, Shanxi University, Taiyuan, Shanxi 030006, China
}

(Received 20 December 2016; revised 15 February 2017; accepted 15 February 2017)

The multiple colliding laser pulse concept formulated by Bulanov et al. (Phys. Rev. Lett., vol. 104, 2010b, 220404) is beneficial for achieving an extremely high amplitude of coherent electromagnetic field. Since the topology of electric and magnetic fields of multiple colliding laser pulses oscillating in time is far from trivial and the radiation friction effects are significant in the high field limit, the dynamics of charged particles interacting with the multiple colliding laser pulses demonstrates remarkable features corresponding to random walk trajectories, limit circles, attractors, regular patterns and Lévy flights. Under extremely high intensity conditions the nonlinear dissipation mechanism stabilizes the particle motion resulting in the charged particle trajectory being located within narrow regions and in the occurrence of a new class of regular patterns made by the particle ensembles.

Key words: plasma dynamics, plasma nonlinear phenomena

\section{Introduction}

Recent progress in laser technology has led to a dramatic increase of laser power and intensity. The lasers are capable of producing electromagnetic field intensities well above $10^{18} \mathrm{~W} \mathrm{~cm}^{-2}$, which corresponds to the relativistic quiver electron energy, and in the near future their radiation may reach intensities of $10^{24} \mathrm{~W} \mathrm{~cm}^{-2}$ and higher (Mourou et al. 2011). As a result, the laser-matter interaction will happen in the radiation friction dominated regimes (Marklund \& Shukla 2006; Mourou, Tajima \& Bulanov 2006; Di Piazza et al. 2012). In a strong electromagnetic field, electrons can be accelerated to such high velocities that the radiation reaction starts

$\dagger$ Email address for correspondence: bulanov.sergei@qst.go.jp 
to play an important role (Zel'dovich 1975; Zhidkov et al. 2002; Bulanov et al. 2004b; Di Piazza 2008; Harvey, Heinzl \& Marklund 2011; Thomas et al. 2012; Heinzl et al. 2015). The radiation friction effects change drastically the laser-plasma interaction leading to fast energy losses (see Koga 2004; Koga, Esirkepov \& Bulanov 2005, 2006) and to phase space contraction, as discussed in Tamburini et al. (2011). Moreover, previously unexplored regimes of the interaction will be entered into, in which quantum electrodynamics (QED) effects such as vacuum polarization, pair production and cascade development can occur (Bell \& Kirk 2008; Di Piazza et al. 2012).

An electromagnetic field intensity of the order of $10^{24} \mathrm{~W} \mathrm{~cm}^{-2}$ can be achieved in the focus of a $1 \mu \mathrm{m}$ wavelength laser of ten petawatt power. For $30 \mathrm{fs}$, i.e. for a ten wave period duration, the laser pulse energy is approximately $300 \mathrm{~J}$. Within the framework of the multiple colliding laser pulses (MCLP) concept formulated in Bulanov et al. (2010b) (see Bulanov et al. (2010a), Gonoskov et al. (2012, 2013), Gelfer et al. (2015) for development of this idea), the laser radiation with given energy $\mathcal{E}_{\text {las }}$ is subdivided into several beams each of them having $1 / N$ of the laser energy, where $N$ is the number of beams. If the beams interfere in the focus in a constructive way, i.e. their electric fields are summed, the resulting electric field and the laser intensity are equal to $E_{N}=\sqrt{N} E_{\text {las }}$ and $I_{N}=N I_{\text {las }}$, respectively. Here $E_{\text {las }}$ and $I_{\text {las }}$ are the electric field and the intensity of the laser light. For a large number of beams there is a diffraction constraint on the electric field amplitude in the focus region. In the limit $N \rightarrow \infty$ the electromagnetic field can be approximated by the three-dimensional dipole configurations (see Bulanov et al. 2010a) for which the electric field maximum is given by (Bassett 1986)

$$
E_{m}=8 \pi \sqrt{\frac{\mathcal{P}_{\text {las }}}{3 c \lambda^{2}}},
$$

where $\mathcal{P}_{\text {las }}, \lambda$ and $c$ are the laser power, wavelength and speed of light in vacuum, respectively.

Since the radiation friction and QED processes both depend on the particle's momentum, the strength of the present electromagnetic field and on their mutual orientation, they are crucial in understanding the dynamics of charged particles in an electromagnetic field in the regime of radiation dominance. Even in the simplest MCLP case, two counter-propagating plane waves, the particle behaviour in the standing wave is quite complicated. It demonstrates regular and chaotic motion, random walk, limit circles and strange attractors as is shown by Mendonca (1983), Bauer, Mulser \& Steeb (1995), Sheng et al. (2002), Lehmann \& Spatschek (2012, 2016), Gonoskov et al. (2014), Bashinov, Kim \& Sergeev (2015), Bulanov et al. (2015), Esirkepov et al. (2015), Jirka et al. (2016), Kirk (2016). As is well known, the standing wave configuration is widely used in classical electrodynamics and in QED theory. This is due to the fact that in the planes where the magnetic field vanishes, the charged particle may be considered to be interacting with an oscillating pure electric field. This provides great simplification of the theoretical description. In addition, as has been noted above, in a standing wave formed by two colliding laser pulses, the resulting electromagnetic (EM) field configuration facilitates QED effects (see Bulanov et al. 2004a, 2006, 2010b). Computer simulations presented in Gonoskov et al. (2016), Gong et al. (2017), Vranic et al. (2017) show that the MCLP concept can be beneficial for realizing such important laser-matter interaction regimes as, for example, the electron-positron pair production via the Breit-Wheeler process (see Vranic et al. 2017) and the high efficiency $\gamma$-ray flash generation due 
to nonlinear Thomson or multi-photon Compton scattering, as shown in Gonoskov et al. (2016), Gong et al. (2017). Another configuration for the generation of a $\gamma$-ray flash is a single laser pulse irradiating an overdense plasma target (see Nakamura et al. 2012; Ridgers et al. 2012; Corvan, Zepf \& Sarri 2016; Levy et al. 2016). The applications of the laser based $\gamma$-ray sources are reviewed in Gales et al. (2016). The radiation friction effects on ion acceleration, on magnetic field self-generation and on high-order harmonics in laser plasmas have been studied in Tamburini et al. (2010, 2011), Liseykina, Popruzhenko \& Macchi (2016) and Tang, Kumar \& Keitel (2016), respectively.

It is not surprising that the dynamics of the electron interacting with three-, four-, etc. colliding pulses is even more complicated and rich with novel patterns.

The present paper contains the theoretical analysis of the electron motion in the standing EM wave generated by two, three and four colliding focused EM pulses. The paper is organized as follows. In the next section we introduce the notations used, describe the field configurations and equations of motion and present the dimensionless parameters characterizing the charged particle interaction with a high intensity EM field. Then, in $\S 3$ we briefly recover the main features of the electron motion in two counter-propagating plane waves. In $\S 4$ we formulate a simple theoretical model of the stabilization of the particle motion in the oscillating field due to nonlinear dissipation effects, which explains the radiative electron trapping revealed earlier in Gonoskov et al. (2012, 2013), Ji et al. (2014), Bulanov et al. (2015), Esirkepov et al. (2015), Jirka et al. (2016), Kirk (2016). Section 5 relates to the regular and chaotic electron motion in three s-polarized laser pulses. The radiating electron dynamics in the four s- and p-polarized colliding EM pulses is discussed in $\S 6$. Section 7 summarizes the conclusions.

\section{Field configurations, dimensionless parameters and equations of motion}

\section{1. $N$ colliding EM waves}

Consider $N$ monochromatic plane waves in vacuum with the same frequencies $\omega_{0}$ and equal amplitudes $a_{n}$. We assume that the wave vectors $\boldsymbol{k}_{n}$ are in the $(x, y)$ plane. The wave vector of the $n_{t h}$ wave is equal to

$$
\boldsymbol{k}_{n}=k_{0}\left[\sin \left(\theta_{n}\right) \boldsymbol{e}_{x}+\cos \left(\theta_{n}\right) \boldsymbol{e}_{y}\right]
$$

where $k_{0}=\omega_{0} / c, \theta_{n}=2 \pi(n-1) / N, n=1,2,3, \ldots, N$ and $\boldsymbol{e}_{x}$ and $\boldsymbol{e}_{y}$ are unit vectors in the $x$ and $y$ directions.

It is convenient to describe the s-polarized EM waves with the electric field normal to the $(x, y)$ plane, i.e. $\boldsymbol{E}=E_{z} \boldsymbol{e}_{z}$ with the unit vector $\boldsymbol{e}_{z}$ along the $z$ direction, in terms of $E_{z}(x, y, t)$ equal to

$$
E_{z}=E_{n} \sum_{n=1}^{N} \sin \left\{\omega_{0}\left[t-\frac{\sin \left(\theta_{n}\right) x-\cos \left(\theta_{n}\right) y}{c}\right]\right\} .
$$

Here the amplitude of the $n_{t h}$ wave is $E_{n}=E_{0} / \sqrt{N}$ where $E_{0}=E_{\text {las }}$. The magnetic field can be expressed by using Maxwell's equations: $(1 / c) \partial_{t} B_{x}=-\partial_{y} E_{z}$ and $(1 / c) \partial_{t} B_{y}=\partial_{x} E_{z}$.

In the case of p-polarized EM waves with the magnetic field normal to the $(x, y)$ plane, $\boldsymbol{B}=B_{z} \boldsymbol{e}_{z}$, the $B_{z}$ field of colliding $N$ pules is given by

$$
B_{z}=B_{n} \sum_{n=1}^{N} \cos \left\{\omega_{0}\left[t-\frac{\sin \left(\theta_{n}\right) x-\cos \left(\theta_{n}\right) y}{c}\right]\right\}
$$


with $B_{n}=E_{\text {las }} / \sqrt{N}$ and the electric field components expressed via Maxwell's equations as $(1 / c) \partial_{t} E_{x}=\partial_{y} B_{z}$ and $(1 / c) \partial_{t} E_{y}=-\partial_{x} E_{z}$, respectively.

\subsection{Dimensionless parameters characterizing interaction of laser radiation with charged particles}

Introducing the normalized variables, we change the space and time coordinates to $x / \lambda \rightarrow x$ and $t \omega / 2 \pi \rightarrow t$.

The interaction of charged particles with intense EM fields is characterized by several dimensionless and relativistic invariant parameters (Nikishov \& Ritus 1964a; Di Piazza et al. 2012; Bulanov et al. 2015).

The first parameter is

$$
a=\frac{e \sqrt{A_{\mu} A^{\mu}}}{m_{e} c^{2}},
$$

where $A^{\mu}$ is the 4-potential of the electromagnetic field with $\mu=0,1,2,3,4$. Here and below summation over repeating indexes is assumed. This parameter is relativistically invariant for a plane EM wave. It is related to the wave normalized amplitude introduced above. When it is equal to unity, i.e. the intensity of a linearly polarized EM wave is $I_{R}=1.37 \times 10^{18}(1 \mu \mathrm{m} / \lambda)^{2} \mathrm{~W} \mathrm{~cm}^{-2}$, the quiver electron motion becomes relativistic.

The ratio, $e E / m_{e} \omega c$, the dimensionless EM field amplitude, measures the work in units of $m_{e} c^{2}$ produced by the field on an electron over the distance equal to the field wavelength. Here, $e$ and $m_{e}$ are the charge and mass of an electron, $E$ and $\omega$ are the EM field strength and frequency and $c$ is the speed of light.

The second dimensionless parameter is $\varepsilon_{\text {rad }}$ :

$$
\varepsilon_{\text {rad }}=\frac{4 \pi r_{e}}{3 \lambda}=1.18 \times 10^{-8}\left(\frac{1 \mu \mathrm{m}}{\lambda}\right),
$$

which is proportional to the ratio of the classical electron radius $r_{e}=e^{2} / m_{e} c^{2}=2.8 \times$ $10^{-13} \mathrm{~cm}$ to the laser radiation wavelength, $\lambda$. It essentially determines the strength of the radiation reaction effects for an electron radiating an EM wave.

When one micron wavelength laser intensities exceed $10^{23} \mathrm{~W} \mathrm{~cm}^{-2}$, the nonlinear quantum electrodynamics effects begin to play a significant role in laser plasma interactions (e.g. see Bulanov et al. (2015) and literature cited therein). These effects manifest themselves through multi-photon Compton and Breit-Wheeler effects (Nikishov \& Ritus 1964a,b; Ritus 1985) (see Narozhnyi \& Fofanov (1996), Boca \& Florescu (2009), Ehlotzky, Krajewska \& Kamiśki (2009), Heinzl, Ilderton \& Marklund (2010a), Heinzl, Seipt \& Kämpfer (2010b), Mackenroth \& Di Piazza (2011), Krajewska \& Kamiński (2012), Titov et al. (2012), Harvey, Heinzl \& Ilderton (2009) for recent studies), i.e. through either photon emission by an electron or positron, or electron-positron pair production by a high energy photon, respectively. The multi-photon Compton and Breit-Wheeler processes are characterized in terms of two dimensionless relativistic and gauge invariant parameters (Nikishov \& Ritus $1964 a)$ :

$$
\chi_{e}=\frac{\sqrt{\left|F^{\mu \nu} p_{v}\right|^{2}}}{E_{S} m_{e} c} \quad \text { and } \quad \chi_{\gamma}=\frac{\lambda_{C} \sqrt{\left|F^{\mu \nu} k_{v}\right|^{2}}}{E_{S}} \text {. }
$$

where $p_{v}$ and $\hbar k_{v}$ denote the 4-momenta of an electron or positron undergoing the Compton process and a photon undergoing the Breit-Wheeler process, the 4-tensor 
of the electromagnetic field is defined as $F_{\mu \nu}=\partial_{\mu} A_{\nu}-\partial_{\nu} A_{\mu}$, with the critical QED electric field

$$
E_{S}=\frac{m_{e}^{2} c^{3}}{e \hbar} .
$$

This field is also known as the 'Schwinger field' (Beresteskii, Lifshitz \& Pitaevskii 1982). Its amplitude is approximately $10^{18} \mathrm{~V} \mathrm{~cm}^{-1}$, which corresponds to the radiation intensity $\approx 10^{29} \mathrm{~W} \mathrm{~cm}^{-2}$. The work produced by the field $E_{S}$ on an electron over the distance equal to the reduced Compton wavelength, $\lambda_{C}=\hbar / m_{e} c=3.86 \times 10^{-11} \mathrm{~cm}$ equals $m_{e} c^{2}$. Here $\hbar$ is the reduced Planck constant.

In three-dimensional (3-D) notation the parameter $\chi_{e}$ given by (2.6) reads

$$
\chi_{e}=\frac{\gamma_{e}}{E_{S}} \sqrt{\left(\boldsymbol{E}+\frac{\boldsymbol{p}_{e} \times \boldsymbol{B}}{m_{e} c \gamma_{e}}\right)^{2}-\left(\frac{\boldsymbol{p}_{e} \cdot \boldsymbol{E}}{m_{e} c \gamma_{e}}\right)^{2}} .
$$

For the parameter $\chi_{\gamma}$ defined by (2.6) we have

$$
\chi_{\gamma}=\frac{\hbar}{E_{S} m_{e} c} \sqrt{\left(\frac{\omega_{\gamma}}{c} \boldsymbol{E}+\boldsymbol{k}_{\gamma} \times \boldsymbol{B}\right)^{2}-\left(\boldsymbol{k}_{\gamma} \cdot \boldsymbol{E}\right)^{2}} .
$$

Here $\gamma_{e}, \boldsymbol{p}_{e}, \omega_{\gamma}$ and $\boldsymbol{k}_{\gamma}$ correspond to the representation of the electron 4-momentum $p_{v}$ and of the photon 4-wavenumber $k_{v}$ as $p_{v}=\left(\gamma_{e} m_{e} c, \boldsymbol{p}\right)$ and $k_{v}=\left(\omega_{\gamma} / c, \boldsymbol{k}_{\gamma}\right)$, respectively. The parameter $\chi_{e}$ can also be defined as the ratio of the electric field to the critical electric field of quantum electrodynamics, $E_{S}$, in the electron rest frame. In particular, it characterizes the probability of the gamma-photon emission by an electron with 4-momentum $p_{v}$ in the field of the electromagnetic wave, in the Compton scattering process.

The parameter $\chi_{\gamma}$ characterizes the probability of electron-positron pair creation by the photon with the momentum $\hbar k_{v}$ interacting with a strong EM wave in the BreitWheeler process.

The probabilities of the Compton scattering and of the Breit-Wheeler processes depend strongly on $\chi_{e}$ and $\chi_{\gamma}$, reaching optimal values when $\chi_{e} \sim 1$ and $\chi_{\gamma} \sim 1$ (Nikishov \& Ritus 1964a).

In the case of an electron interaction with a plane EM wave propagating along the $x$-axis with phase and group velocity equal to speed of light in vacuum the parameters of the interaction can be written in terms of EM field strength, normalized by the QED critical field given by (2.7), and either the electron $\gamma_{e}$-factor or the photon energy $\hbar \omega_{\gamma}$ :

$$
\chi_{e}=\frac{E}{E_{S}}\left(\gamma_{e}-\frac{p_{x}}{m_{e} c}\right)
$$

and

$$
\chi_{\gamma}=\frac{E}{E_{S}} \frac{\hbar\left(\omega_{\gamma}-k_{\gamma, x} c\right)}{m_{e} c^{2}} .
$$

For an electron interacting with the EM wave the linear combination of the electron energy and momentum,

$$
h_{e}=\gamma_{e}-p_{x} / m_{e} c
$$

on the right-hand side of (2.10) is an integral of motion (Landau \& Lifshitz 1982). Its value is determined by initial conditions. 
(a)

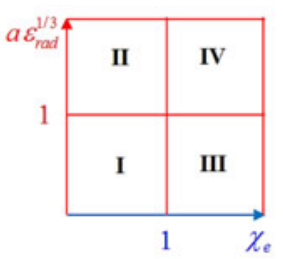

(b)

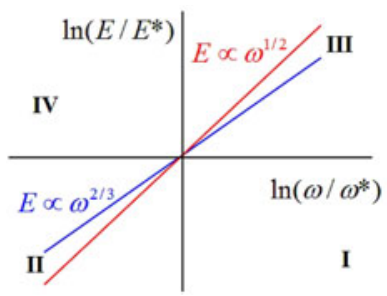

FIGURE 1. Regimes of electromagnetic field interaction with matter on the plane of parameters: $(a)$ the normalized EM wave amplitude $a \varepsilon_{\text {rad }}^{1 / 3}$ and the parameter $\chi_{e}$; (b) accordingly the $\left(\ln \left(E / E^{*}\right), \ln \left(\omega / \omega^{*}\right)\right)$ plane, where $E^{*}$ and $\omega^{*}$ are given by (2.14) and (2.15), respectively. The parameter planes are subdivided into 4 domains: (I) electron - EM field interaction in the particle dominated radiation reaction domain; (II) electron - EM field interaction is dominated by the radiation reaction; (III) electron - EM field interaction is in the particle dominated QED regime; (IV) electron - EM field interaction is in the radiation dominated QED regime.

If an electron/positron or a photon co-propagates with the EM wave, then in the former case the parameter $\chi_{e}$ is suppressed by a factor $\left(2 \gamma_{e, 0}\right)^{-1}$, i.e. $\chi_{e} \simeq\left(2 \gamma_{e, 0}\right)^{-1}\left(E / E_{S}\right)$, where $\gamma_{e, 0}$ is the electron gamma-factor before interaction with the laser pulse. In the later case, when the gamma-photon co-propagates with the EM wave, the parameter $\chi_{\gamma}$ is equal to zero, $\chi_{\gamma}=0$, because $\omega_{\gamma}=k_{\gamma, x} c$. On the contrary, the parameter $\chi_{e}$ can be enhanced to approximately $2 \gamma_{e, 0} E / E_{S}$, when the electron interacts with a counter-propagating laser pulse. Therefore the head-on collision configuration has an apparent advantage for strengthening the electron-EM wave interaction and, in particular, for enhancing the $\gamma$-ray production due to nonlinear Thomson or/and Compton scattering.

Depending on the energy of charged particles and field strength the interaction happens in one of the following regimes parametrized by the values of $a, \chi_{e}$ and $\chi_{\gamma}$ :

(i) $a>1$, the relativistic interaction regime (Mourou et al. 2006);

(ii) $a>\varepsilon_{\text {rad }}^{-1 / 3}$, the interaction becomes radiation dominated (Zhidkov et al. 2002; Bulanov et al. 2004b; Bashinov \& Kim 2013);

(iii) $\chi_{e} \geqslant 1$ the quantum effects begin to manifest themselves (Di Piazza, Hatsagortsyan \& Keitel 2010; Bulanov et al. 2011a, 2015); and

(iv) $\chi_{e}>1, \chi_{\gamma}>1$ marks the condition for the EM avalanche (Bulanov et al. 2010a; Fedotov et al. 2010; Elkina et al. 2011; Nerush et al. 2011; Bulanov et al. 2013), which is the phenomenon of exponential growth of the number of electron-positrons and photons in the strong EM field, being able to develop. These conditions can be supplemented by $\alpha a>1$, which indicates that the number of photons emitted incoherently per laser period can be larger than unity as has been noted by Di Piazza et al. (2010). Here the parameter $\varepsilon_{\text {rad }}$ is given by (2.5) and $\alpha=e^{2} / \hbar c \approx 1 / 137$ is the fine structure constant.

As one can see two dimensionless parameters, $a$ and $\chi_{e}$, can be used to subdivide the $\left(a, \chi_{e}\right)$ plane into four domains shown in figure 1(a) (see also Bulanov et al. 2015; Bulanov 2017). The $\chi_{e}=1$ line divides the plane into the radiation reaction description of the interaction domain $\left(\chi_{e}<1\right)$ and QED description of interaction domain $\left(\chi_{e}>1\right)$. The $a=\varepsilon_{\text {rad }}^{-1 / 3}$ line divides the plane into radiation dominated $\left(a>\varepsilon_{\text {rad }}^{-1 / 3}\right)$ and particle dominated $\left(a<\varepsilon_{\text {rad }}^{-1 / 3}\right)$ regimes of interaction domains. We note that the $a=\varepsilon_{\text {rad }}^{-1 / 3}$ threshold comes from the requirement for an electron to emit the amount of energy 
per EM wave period equal to the energy gain from the EM wave during the wave period. If one takes into account the discrete nature of the photon emission, then the same condition will take the form $a m_{e} c^{2}=\hbar \omega_{\gamma}\left(\lambda / L_{R}\right)$ (Ritus 1985), where $L_{R}$ is the radiation length is of the order of $2 \lambda / a$ for $\chi_{e} \ll 1$ and $\lambda \gamma_{e}^{1 / 3} / a^{2 / 3}$ for $\chi_{e} \gg 1$ (Nikishov \& Ritus 1964a,b; Bolotovskii \& Voskresenskii 1966; Ritus 1985). This condition in the limit $\chi_{e} \rightarrow 0$ tends to the classical limit $a=\varepsilon_{\text {rad }}^{-1 / 3}$.

The intersection point, where $a_{\text {rad }}=\varepsilon_{\text {rad }}^{-1 / 3}$ and the parameter $\chi_{e}$ is equal to unity, determines critical values of the EM wave amplitude $\varkappa_{a} a^{*}$ with

$$
a^{*}=\left(\frac{3 c}{2 r_{e} \omega^{*}}\right)^{1 / 3}=\frac{\hbar c}{e^{2}}=\frac{1}{\alpha},
$$

i.e. the wave electric field is $\varkappa_{a} \varkappa_{\omega} E^{*}$, where

$$
E^{*}=E_{S} \alpha
$$

and the wave frequency $\varkappa_{\omega} \omega^{*}$ with $\omega^{*}$ given by

$$
\omega^{*}=\frac{e^{4} m_{e}}{\hbar^{3}}=\frac{m_{e} c^{2}}{\hbar \alpha^{2}} .
$$

Here $\alpha=1 / 137$ is the fine structure constant and $\varkappa_{a}$ and $\varkappa_{\omega}$ are constants of the order of unity. The normalized EM wave amplitude equals $a^{*}=137$ with corresponding wave intensity $I^{*}=2.6 \times 10^{22} \mathrm{~W} \mathrm{~cm}^{-2}$. The corresponding photon energy is $\hbar \omega^{*}=$ $m_{e} c^{2} / \alpha^{2} \approx 27 \mathrm{eV}$. We note that the value of $a^{*}=1 / \alpha$ corresponds to one of the conditions for the charged particle interaction with the EM field to be in the QED regime, $\alpha a>1$ (see also Di Piazza et al. 2010).

Concrete values of the coefficients $\varkappa_{a}$ and $\varkappa_{\omega}$ depend on the specific electromagnetic configuration. For example, in the case of a rotating homogeneous electric field (which can be formed in the antinodes of an electric field in the standing EM wave) analysed in Bulanov et al. (2015), they are $\varkappa_{a}=3$ and $\varkappa_{\omega}=1 / 18$, respectively, which gives $\varkappa_{a} a^{*}=411$, with the intensity equal to $2.3 \times 10^{23} \mathrm{~W} \mathrm{~cm}{ }^{-2}$, and $\varkappa_{\omega} \hbar \omega^{*}=m_{e} c^{2} \alpha^{2} / 18 \approx$ $1.5 \mathrm{eV}$.

Here we would like to attract attention to the relationship between the well-known critical electric field of classical electrodynamics $E_{c r}$, the critical electric field of quantum electrodynamics $E_{S}$ and the electric field $E^{*}$. They can be written as $E_{c r}=e / r_{e}^{2}, E_{S}=e / r_{e} \lambda_{C}$ and $E^{*} \approx e / \lambda_{C}^{2}$, respectively. In other words we have $E_{S}=E_{c r} \alpha$ and $E^{*}=E_{c r} \alpha^{2}$.

Using the relationships obtained above we find that on the line $a \varepsilon_{r a d}^{1 / 3}=1$ the wave electric field is proportional to the frequency in the $2 / 3$ power, i.e. $E / E^{*}=\left(\omega / \omega^{*}\right)^{2 / 3}$, and on the line $\chi_{e}=1$ we have $E / E^{*}=\left(\omega / \omega^{*}\right)^{1 / 2}$.

Figure $1(b)$ shows the $\left(\ln \left(E / E^{*}\right), \ln \left(\omega / \omega^{*}\right)\right)$ plane with 4 domains. The lines intersect each other at the point $(0,0)$, i.e. at the point where $E=E^{*}$ and $\omega=\omega^{*}$.

\subsection{Radiation friction force with the QED form factor}

In order to describe the relativistic electron dynamics in the electromagnetic field we shall use the equations of electron motion:

$$
\begin{gathered}
\frac{\mathrm{d} \boldsymbol{p}}{\mathrm{d} t}=e\left(\boldsymbol{E}+\frac{\boldsymbol{v}}{c} \times \boldsymbol{B}\right)+F_{r a d}, \\
\frac{\mathrm{d} \boldsymbol{x}}{\mathrm{d} t}=\frac{\boldsymbol{p}}{m_{e} \gamma},
\end{gathered}
$$


where the radiation friction force, $F_{\text {rad }}=G_{e} f_{\text {rad }}$, is the product of the classical radiation friction force, $\boldsymbol{f}_{\text {rad }}$, in the Landau-Lifshitz form (Landau \& Lifshitz 1982):

$$
\begin{aligned}
\boldsymbol{f}_{\text {rad }}= & \frac{2 e^{3}}{3 m_{e} c^{3} \gamma}\left\{\partial_{t}+(\boldsymbol{v} \nabla) \boldsymbol{E}+\frac{1}{c}\left[\boldsymbol{v} \times\left(\partial_{t}+(\boldsymbol{v} \nabla) \boldsymbol{B}\right]\right\}\right. \\
& +\frac{2 e^{4}}{3 m_{e}^{2} c^{4}}\left\{\boldsymbol{E} \times \boldsymbol{B}+\frac{1}{c}[\boldsymbol{B} \times(\boldsymbol{B} \times \boldsymbol{v})+\boldsymbol{E}(\boldsymbol{v} \cdot \boldsymbol{E})]\right\} \\
& -\frac{2 e^{4}}{3 m_{e}^{2} c^{5}} \gamma^{2} \boldsymbol{v}\left\{\left(\boldsymbol{E}+\frac{1}{c} \boldsymbol{v} \times \boldsymbol{B}\right)^{2}-\frac{1}{c^{2}}(\boldsymbol{v} \cdot \boldsymbol{E})^{2}\right\}
\end{aligned}
$$

and a form factor $G_{e}$, which takes into account the quantum electrodynamics weakening of the radiation friction (Sokolov, Klepikov \& Ternov 1952; Schwinger 1954; Erber 1966; Beresteskii et al. 1982; Sokolov et al. 2010). Discussions of the relationship between the Landau-Lifshitz and Lorentz-Abraham-Dirac forms of the radiation friction force and what form of the force follows from the QED calculation, can be found in Bulanov et al. (2011b), Ilderton \& Torgrimsson (2013), Zhang (2013) and in the literature cited therein.

As we have noted above, the threshold of the QED effects is determined by the dimensionless parameter $\chi_{e}$ given by (2.8). For example, if an electron moves in the magnetic field $B$, the parameter is equal to $\chi_{e} \approx \gamma_{e}\left(B / B_{S}\right)$, where $B_{S}=m_{e}^{2} c^{3} / e \hbar$ is the QED critical magnetic field (see also (2.7)). The energy of the emitted synchrotron photons is

$$
\hbar \omega_{\gamma}=m_{e} c^{2} \gamma_{e} \frac{\chi_{e}}{2 / 3+\chi_{e}} .
$$

In the limit $\chi_{e} \ll 1$ the frequency $\omega_{\gamma}$ is equal to $(3 / 2) \omega_{B e} \gamma_{e}^{2}$ in accordance with classical electrodynamics (see Landau \& Lifshitz 1982). Here $\omega_{B e}=e B / m_{e} c$ is the Larmor frequency. If $\chi_{e} \gg 1$ the photon energy is equal to the energy of the radiating electron: $\hbar \omega_{\gamma}=m_{e} c^{2} \gamma_{e}$.

The radiation friction force in the limit $\gamma_{e} \rightarrow \infty$, i.e. the last term on the right-hand side of (2.18) retained, can be written in the following form (see also Sokolov et al. (1952), Schwinger (1954), Erber (1966), Sokolov et al. (2010), Bulanov et al. (2015) and literature cited therein)

$$
\boldsymbol{f}_{\text {rad }}=-\frac{2 \alpha c G_{e}\left(\chi_{e}\right) \chi_{e}^{2}}{3 \lambda_{C}} \boldsymbol{p} .
$$

Here the QED effects are incorporated into the equations of the electron motion by using the form factor $G_{e}\left(\chi_{e}\right)$ (see Sokolov et al. 1952), which is equal to the ratio of full radiation intensity to the intensity of the radiation emitted by a classical electron. It reads

$$
G_{e}\left(\chi_{e}\right)=\frac{3}{4} \int_{0}^{\infty}\left[\frac{4+5 \chi_{e} x^{3 / 2}+4 \chi_{e}^{2} x^{3}}{\left(1+\chi_{e} x^{3 / 2}\right)^{4}}\right] \Phi^{\prime}(x) x \mathrm{~d} x,
$$

where $\Phi(x)$ is the Airy function (Abramovitz \& Stegun 1964). In (2.20) we neglect the effects of the discrete nature of the photon emission in quantum electrodynamics (see Duclous, Kirk \& Bell 2011; Brady et al. 2012; Thomas et al. 2012; Bulanov et al. 2013; Bashinov et al. 2015; Esirkepov et al. 2015; Jirka et al. 2016). 
In the limit $\chi_{e} \ll 1$ the form factor $G\left(\chi_{e}\right)$ tends to unity as

$$
\begin{aligned}
G_{e}\left(\chi_{e}\right) & =1-\frac{55 \sqrt{3}}{16} \chi_{e}+48 \chi_{e}^{2}+\cdots \\
& \approx 1-5.95 \chi_{e}+48 \chi_{e}^{2}+\cdots
\end{aligned}
$$

For $\chi_{e} \gg 1$ it tends to zero as

$$
\begin{aligned}
G_{e}\left(\chi_{e}\right) & =\frac{32 \pi}{273^{5 / 6} \Gamma(1 / 3) \chi_{e}^{4 / 3}}-\frac{1}{\chi_{e}^{2}}+\cdots \\
& \approx \frac{0.5564}{\chi_{e}^{4 / 3}}-\frac{1}{\chi_{e}^{2}}+\cdots
\end{aligned}
$$

However, expression (2.21) and the asymptotical dependences (2.22) and (2.23) are not convenient for implementation in computer codes. For the sake of calculation simplicity we shall use the following approximation

$$
G_{R}\left(\chi_{e}\right) \approx \frac{1}{\left(1+8.93 \chi_{e}+2.41 \chi_{e}^{2}\right)^{2 / 3}} .
$$

Within the interval $0<\chi_{e}<10$ the accuracy of this approximation is better than $1 \%$.

\section{Electron motion in the standing EM wave formed by two counter-propagating EM pulses}

\subsection{EM field configuration}

An electron interaction with an EM field formed by two counter-propagating waves has been addressed a number of times in high field theory using classical quantum electrodynamics approaches because it provides one of the basic EM configurations where important properties of a radiating electron can be revealed (e.g. see above cited publications Mendonca 1983; Di Piazza et al. 2012; Lehmann \& Spatschek 2012, 2016; Gonoskov et al. 2013, 2014; Bashinov et al. 2015; Bulanov et al. 2015; Chang et al. 2015; Esirkepov et al. 2015; Lobet et al. 2015; Bashinov, Kumar \& Kim 2016; Grismayer et al. 2016; Jirka et al. 2016; Kirk 2016). Here, we present the results of the analysis of electron motion in a standing EM wave in order to compare them below with the radiating electron behaviour in a more complicated EM configuration formed by three or four waves with various polarizations.

Here we consider an electron interaction with the electromagnetic field corresponding to two counter-propagating linearly polarized waves of equal amplitude, $\left(a_{0} / 2\right) \cos (t+x)$ and $\left(a_{0} / 2\right) \cos (t-x)$, forming a standing wave. The field is given by the electromagnetic 4-potential

$$
\boldsymbol{A}=a_{0} \cos t \cos x \boldsymbol{e}_{z}
$$

This is a standing electromagnetic wave with zero magnetic and electric field nodes located at the coordinates $x= \pm \pi n$ and $x= \pm \pi(n+1 / 2)$ with $n=0,1,2, \ldots$, respectively.

Numerical integration of the electron motion equations with the radiation friction force in the form (2.20) shows different features of the electron dynamics depending on the electromagnetic wave amplitude and the dissipation parameter $\varepsilon_{\text {rad }}$. 
(a) $z$

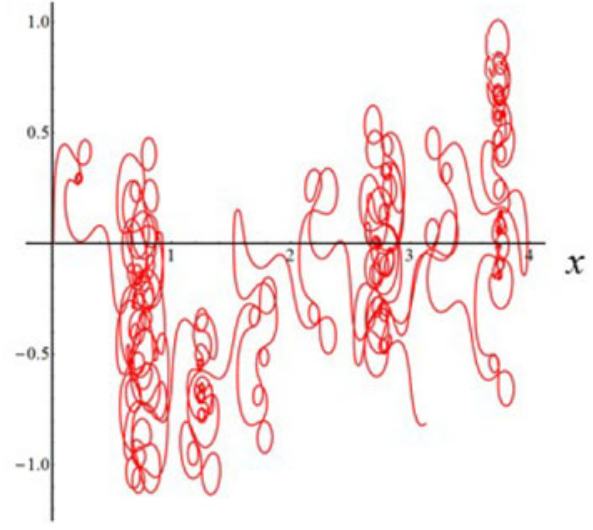

(c) $\gamma_{\mathrm{e}}$

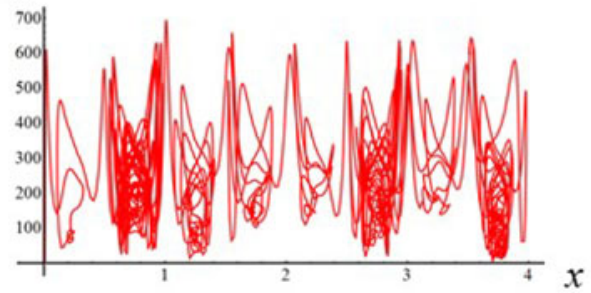

(b)

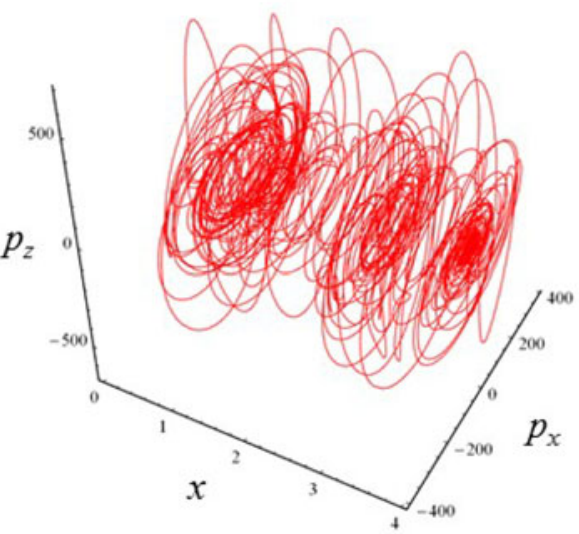

(d) $\chi$

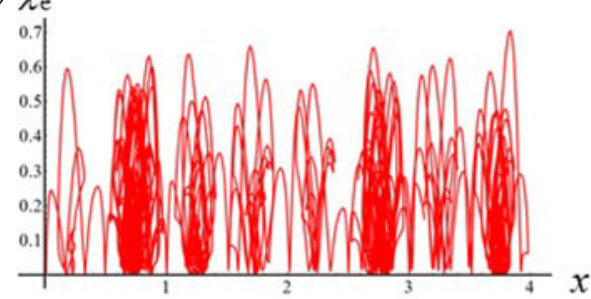

FIGURE 2. (a) Electron trajectories in the $(x, z)$ plane for initial conditions: $x(0)=0.01$, $z(0)=0, p_{x}(0)=0, p_{z}(0)=0$. (b) Trajectory in the phase space $x, p_{x}, p_{z}$; (c) electron gamma-factor, $\gamma_{e}$, versus the coordinate $x ;(d)$ parameter $\chi_{e}$ versus the coordinate $x$, for the same initial conditions. The electromagnetic field amplitude is $a_{0}=617$ and the dissipation parameter is $\varepsilon_{\text {rad }}=1.2 \times 10^{-8}$. The coordinates, time and momentum are measured in $2 \pi c / \omega, 2 \pi / \omega$ and $m_{e} c$ units.

\subsection{Relatively weak intensity limit}

In the limit of relatively weak dissipation, which corresponds to domain I in figure 1, the electron trajectory wanders in the phase space and in the coordinate space as shown in figure 2. In this case the wave amplitude is $a_{0}=618$. The dissipation parameter equals $\varepsilon_{\text {rad }}=2 \times 10^{-8}$. The normalized critical QED field is $a_{S}=e E_{S} / m_{e} \omega c=m_{e} c^{2} / \hbar \omega=4 \times 10^{5}$. The parameter values correspond to the vicinity of the point $\left(a / a^{*}=1, \omega / \omega^{*}=1\right)$ in figure $1(b)$. The integration time equals 75 .

Figure 2 demonstrates a typical behaviour of the electron in the limit of relatively low EM wave amplitude. Figure $2(a, b)$ shows that the electron performs a randomwalk-like motion for a long time, being intermittently trapped and untrapped in the vicinities of the zero electric field nodes, where the electric field vanishes. For this parameter choice the equilibrium trajectory at the electric field antinodes is unstable according to Bulanov et al. (2010a) (see also Gong et al. 2016). The maximum value of the electron gamma-factor, $\gamma_{e}$, whose dependence on the coordinate $x$ is plotted in figure 2(c), reaches 700. In an oscillating electric field of amplitude $a=618$ it would be equal to 618 . The parameter $\chi_{e}$ (see figure $2 d$ ) changes between zero and approximately 0.7 , which corresponds, within an order of magnitude, to $\left(a_{0} / a_{S}\right) \gamma_{e}$. 


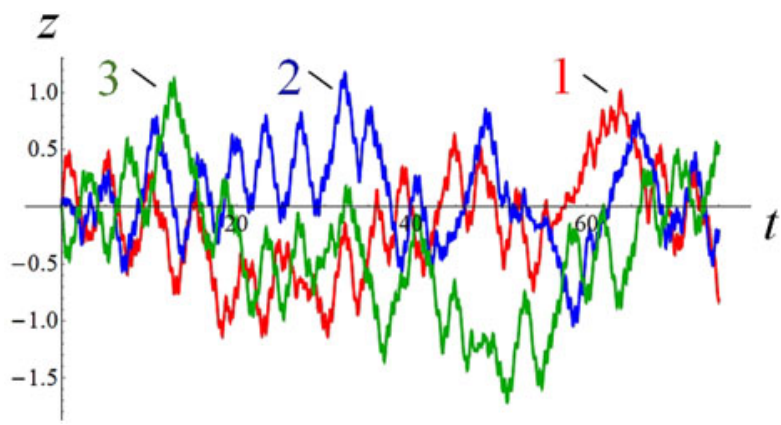

FIGURE 3. Electron coordinate $z$ versus time for initial coordinates $x(0)=0.01-1,0.2-2$, $0.49-3$, other parameters are the same as in figure 2 .

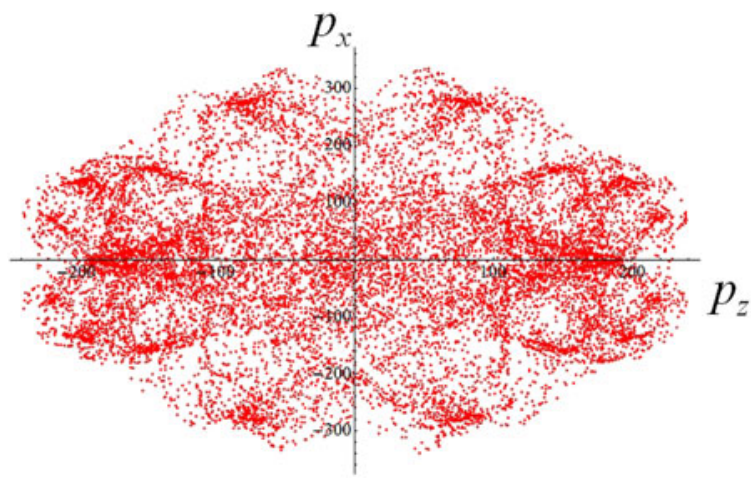

FIgURE 4. The Poincaré sections showing the particle positions in the phase plane $\left(p_{x}, p_{z}\right)$ at discrete times with the time step equal to the period of the driving force. The parameters are the same as in figure 2 for $x(0)=0.01$.

The particle coordinates $z$ versus time in figure 3 , for initial coordinates $x(0)=0.01-1$, $0.2-2,0.49-3$ with other parameters the same as in figure 2 , show its wandering along the coordinate $z$. The particle over-leaping from one field period to another with small-scale oscillations in between seen in figure 3 may correspond to Lévy flights (see Lévy 1954; Metzler \& Klafter 2000; Zaslavsky 2002; Metzler et al. 2007).

Figure 4 shows the Poincare section for the motion of the particle with $x(0)=0.01$ positions in the phase plane $\left(p_{x}, p_{z}\right)$ at discrete times with the time step equal to the period of the driving force. The parameters are the same as in figure 2. The Poincare section demonstrates that this process is stochastic.

\subsection{Random walk}

Now we analyse the time dependence of the random walk, assuming that the particle coordinates $x(t)$ and $z(t)$ are random variables, i.e. the particle displacement in the $(x, z)$ plane equal to $r=\sqrt{x^{2}+z^{2}}$ is also a random variable. As is known in statistics the behaviour of the random variable $f$ is characterized by the expectation $\mu=E[f]$ and variance $\sigma^{2}=\operatorname{Var}[f]$ defined as

$$
E[f]=\lim _{t \rightarrow \infty} \frac{1}{t} \int^{t} f(t) \mathrm{d} t
$$




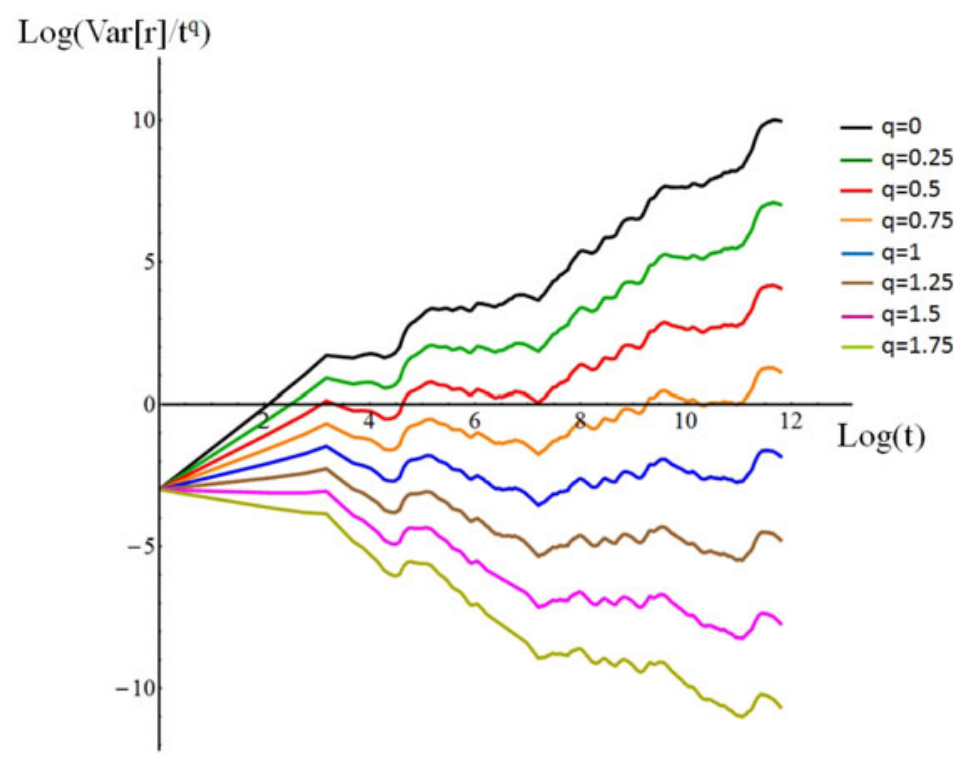

FIgURE 5. Dependences of $\log \left(\operatorname{Var}[r] / t^{q}\right)$ on $\log (t)$ for $0<q<1.25$ for the parameters corresponding to figure 2 .

and

$$
\operatorname{Var}[f]=E\left[(f-E[f])^{2}\right] .
$$

The definition of an expectation in the form (3.2) implies that the probability density function is taken to be a continuous uniform distribution equal to $1 / t$ within the interval $[0, t]$. We assume here that the ergodicity of the processes is expected.

If the random walk process is a Wiener process, which is also called 'Brownian motion', the variance of the walker's coordinate $r(t)$ is proportional to time (e.g. see Durrett 1991). To examine whether or not the random walk seen in figures 2 and 3 is a Wiener process we plot in figure 5 the dependences of $\log \left(\operatorname{Var}[r] / t^{q}\right)$ on $\log (t)$ for $0<q<1.75$. For a Wiener process the parameter $q$ should be equal to 1 . As we can see, in our case of a random walk process the variance is proportional to $t^{q}$ with $q \approx 1$.

\subsection{Moderate intensity regime}

The situation qualitatively changes when the dissipation becomes more significant. In figure 1 this corresponds to the domain II. This case is illustrated in figure 6 , for which the radiation friction parameter is $\varepsilon_{\text {rad }}=6 \times 10^{-9}$, the normalized critical QED field is $a_{S}=8 \times 10^{5}$ and the normalized laser field equals $a_{0}=778$. In figure 6 we present three trajectories for particles with initial conditions: $x(0)=0.01, z(0)=0$, $p_{x}(0)=0, p_{z}(0)=0(\mathrm{red}) ; x(0)=0.2, z(0)=0, p_{x}(0)=0, p_{z}(0)=0$ (blue); $x(0)=0.49$, $z(0)=0, p_{x}(0)=0, p_{z}(0)=0$ (green). As seen in figure $6(a)$, where the trajectories in the $x, z$ plane are shown, independent of the initial position all three trajectories end up in the vicinity of the plane $x=0.25$. Here the EM wave electric field vanishes.

At the coordinate $x=0.25$ the ponderomotive potential has a minimum. It is defined as

$$
\Pi(x)=\frac{1}{2 \pi} \int_{-\pi}^{\pi}\left(\sqrt{1+A(x, t)^{2}}-1\right) \mathrm{d} t
$$


(a) $z, \Pi$

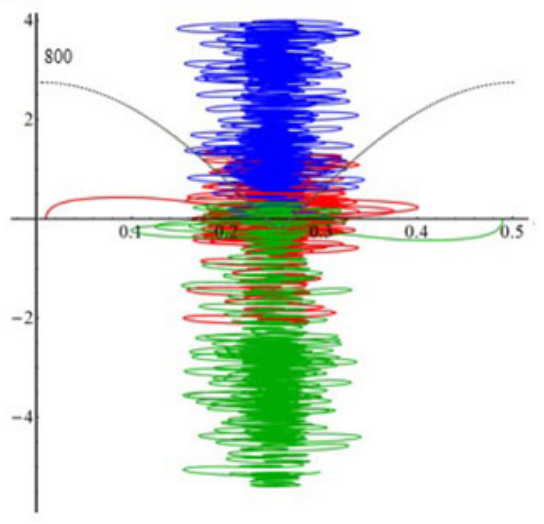

(c)

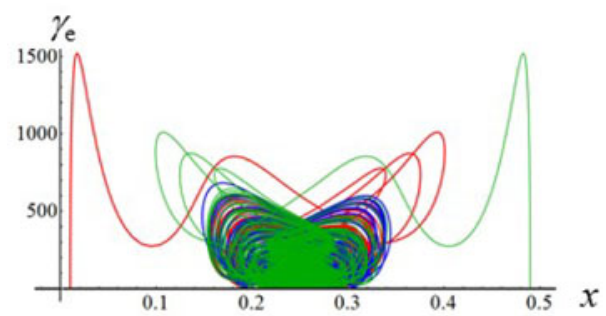

(b)

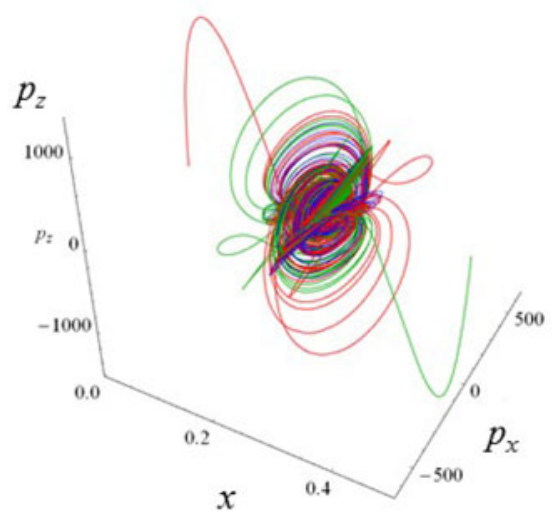

(d) $\chi_{\mathrm{e}}$

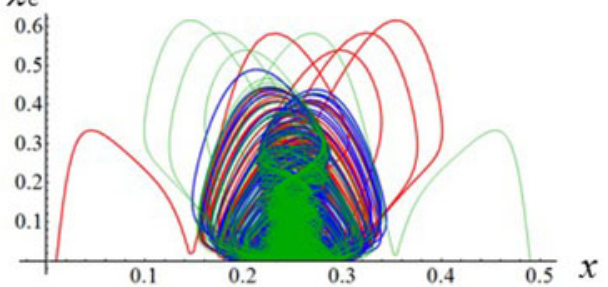

FIGURE 6. Electron motion in the standing EM wave for $\varepsilon_{\text {rad }}=6 \times 10^{-9}, a_{S}=8 \times 10^{5}$ and $a_{0}=778$ for initial conditions: $x(0)=0.01, z(0)=0, p_{x}(0)=0, p_{z}(0)=0$ (red); $x(0)=0.2$, $z(0)=0, p_{x}(0)=0, p_{z}(0)=0$ (blue); $x(0)=0.49, z(0)=0, p_{x}(0)=0, p_{z}(0)=0$ (green). (a) Trajectory in the $x, z$ plane. Dashed line is the ponderomotive potential (3.4) versus the $x$ coordinate; $(b)$ electron trajectories in the $\left(x, p_{x}, p_{z}\right)$ space. $(c)$ Electron gamma-factor, $\gamma_{e}$, versus the coordinate $x$. (d) Parameter $\chi_{e}$ versus the coordinate $x$, for the same initial conditions.

with the vector potential $A(x, t)$ given by (3.1). The dashed curve in figure $6(a)$ presents the ponderomotive potential (3.4) dependence on the $x$ coordinate. In figure $6(b)$ electron trajectories in the $\left(x, p_{x}, p_{z}\right)$ space show the attractors, which have been analysed in detail in Esirkepov et al. (2015) (see figure 7, where the Poincaré section is presented for this case). Electron gamma-factor, $\gamma_{e}$, versus the coordinate $x$ presented in figure $6(c)$ correspond to the case when the dissipation limits the particle energy, which does not exceed the value determined by the amplitude of the EM wave being of the order of $a$. Since the parameter $\chi_{e}(x)$ plotted in figure $6(d)$ is less than unity for all three trajectories, the equation of electron motion with the radiation friction force is still valid for this parameter range.

In figure 7 , we plot the Poincaré section for the particle with the same parameters as in figure 6 for $x(0)=0.01$. Here, the particle positions are in the phase plane $\left(x, p_{x}\right)$ at discrete times with the time step equal to the period of the driving force. The map pattern corresponds to the stochastic regime developed in the particle motion.

\subsection{High intensity regime}

If we choose the parameters in a such the way that the dissipation becomes even more significant, when we approach domain IV in figure 1, the particle behaviour becomes counterintuitive, as can be seen in figure 8 , for which the radiation friction 


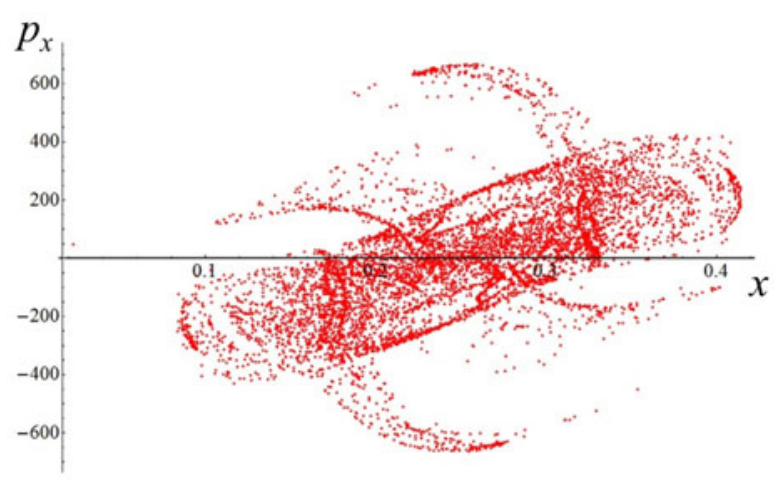

FIgURE 7. The Poincare sections showing the particle positions in the phase plane $\left(x, p_{x}\right)$ at discrete times with the time step equal to the period of the driving force. The parameters are the same as in figure 6 for $x(0)=0.01$.

parameter is $\varepsilon_{\text {rad }}=1.2 \times 10^{-9}$, the normalized critical QED field is $a_{S}=4 \times 10^{6}$ and the normalized laser field equals $a_{0}=1996$. There we present three electron trajectories for the same initial conditions as in figure 4: $x(0)=0.01, z(0)=0$, $p_{x}(0)=0, p_{z}(0)=0(\mathrm{red}) ; x(0)=0.2, z(0)=0, p_{x}(0)=0, p_{z}(0)=0$ (blue); $x(0)=0.49$, $z(0)=0, p_{x}(0)=0, p_{z}(0)=0$ (green). Trajectories in the $x, z$ plane (figure $6 a$ ) are principally different depending on where the particle has been initially located. For $x(0)=0.2$ the trajectory remains in the vicinity of the ponderomotive potential minimum similarly to the case discussed above (figure 6). The dashed line is the ponderomotive potential (3.4) versus the $x$ coordinate. In contrast, particles with initial coordinates near the maximum of the ponderomotive potential are trapped there (similar behaviour was noted in Gonoskov et al. (2014)).

In figure $8(b)$ the electron trajectories in the $\left(x, p_{x}, p_{z}\right)$ space show behaviour typical for limit circles and attractors. The inset shows the zoomed trajectory for $x(0)=0.2$ corresponding to a strange attractor (Esirkepov et al. 2015). The trajectories with $x(0)=0.01$ and $x(0)=0.49$ demonstrate regular limit circles. It follows from figure $8(c)$ that the electron gamma-factor, $\gamma_{e}$, has a moderate value for the electron trapped near the ponderomotive potential minimum (the inset shows zoomed $\gamma_{e}(x)$ for $x(0)=0.2$, and the particles are efficiently accelerated when they are trapped in the region at the ponderomotive potential maximum. For the parameters chosen, $\chi_{e}(x)$ plotted in figure $8(d)$ remains less than unity for all three trajectories, i.e. the QED effects are finite but relatively weak.

In figure 9, we plot the Poincaré section showing the particle with $x(0)=0.49$ position in the phase plane $\left(x, p_{x}\right)$ at discrete times with the time step equal to the period of the driving force. The parameters are the same as in figure 8 for $x(0)=0.49$. Although the map pattern is complicated it does not contain curve broadening, i.e. does not indicate a stochastic regime of the particle motion.

In the next section we discuss the mechanism of dissipative particle trapping in the vicinity of the ponderomotive potential maximum which can explain the observed effects (see also Fedotov et al. 2014 and Esirkepov \& Bulanov 2017).

\section{Simple model of the stabilization of the particle motion in an oscillating field due to the nonlinear friction}

Let us consider a particle motion in a fast oscillating field in a way similar to Landau \& Lifshitz (1976). As in Landau \& Lifshitz (1976) for the sake of simplicity 
(a) $z, \Pi$

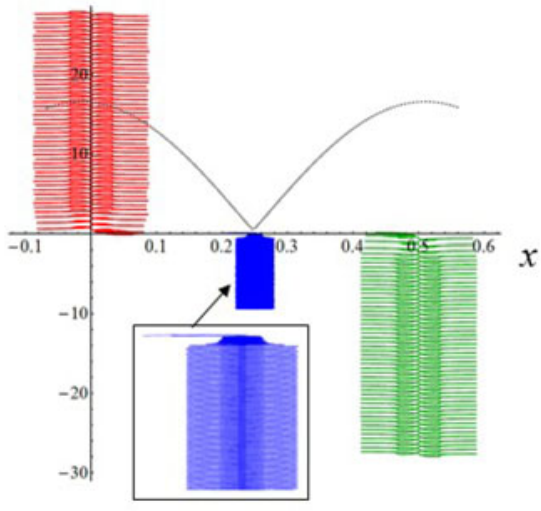

(c)

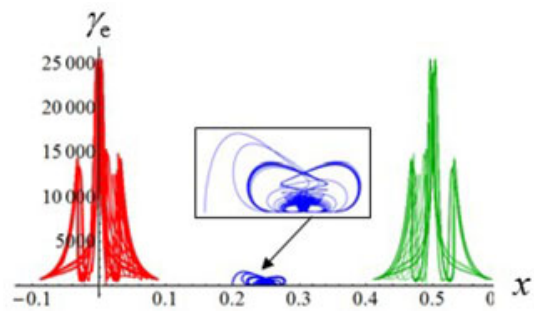

(b)

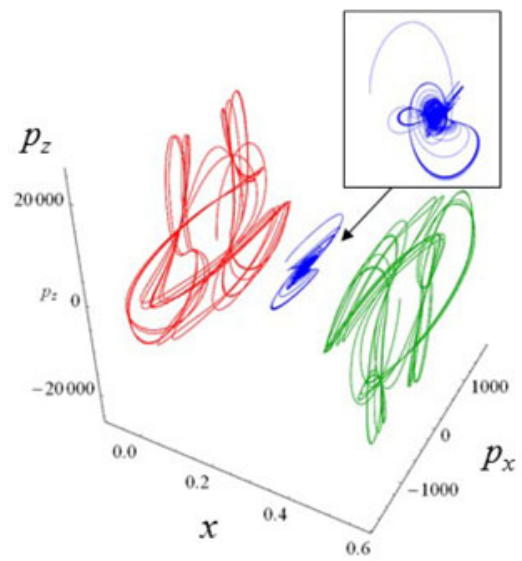

(d)

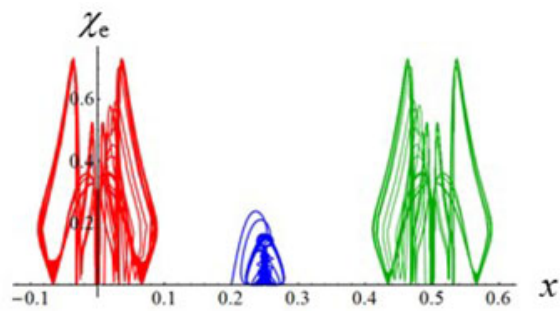

FIGURE 8. Electron motion in the standing EM wave for $\varepsilon_{\text {rad }}=1.2 \times 10^{-9}, a_{S}=4 \times 10^{6}$, $a=1996$ for initial conditions: $x(0)=0.01, z(0)=0, p_{x}(0)=0, p_{z}(0)=0$ (red); $x(0)=0.2$, $z(0)=0, p_{x}(0)=0, p_{z}(0)=0$ (blue); $x(0)=0.49, z(0)=0, p_{x}(0)=0, p_{z}(0)=0$ (green). (a) Trajectory in the $x, z$ plane. Inset shows zoomed trajectory for $x(0)=0.2$. Dashed line is the ponderomotive potential (3.4) versus the $x$ coordinate; $(b)$ electron trajectories in the $\left(x, p_{x}, p_{z}\right)$ space. Inset shows zoomed trajectory for $x(0)=0.2$ corresponding to a strange attractor (Esirkepov et al. 2015). (c) Electron gamma-factor, $\gamma_{e}$, versus the coordinate $x$. Inset shows zoomed $\gamma_{e}(x)$ for $x(0)=0.2$. (d) Parameter $\chi_{e}$ versus the coordinate $x$, for the same initial conditions.

of calculations we assume non-relativistic electron motion in one dimension, where the force acting on the particle depends on the coordinate $x$ and time $t$. In contrast to the consideration in Landau \& Lifshitz (1976), we take into account the effects of the friction. The equation of particle motion is

$$
\ddot{x}+\kappa(F) \dot{x}=F .
$$

Here a dot stands for the time derivative and $\kappa(F)$ is the friction coefficient. It is assumed to depend on the rapidly oscillating driving force,

$$
F(x, t)=f_{1}(x) \cos \omega t+f_{2}(x) \sin \omega t .
$$

We seek a solution of (4.1), assuming that it can be written as a sum of two parts,

$$
x(t)=X(t)+\xi(t)
$$

where $X(t)$ slowly varies with time and $\xi(t)$ is a small fast oscillating periodic function, $|\xi| \ll|X|$. 


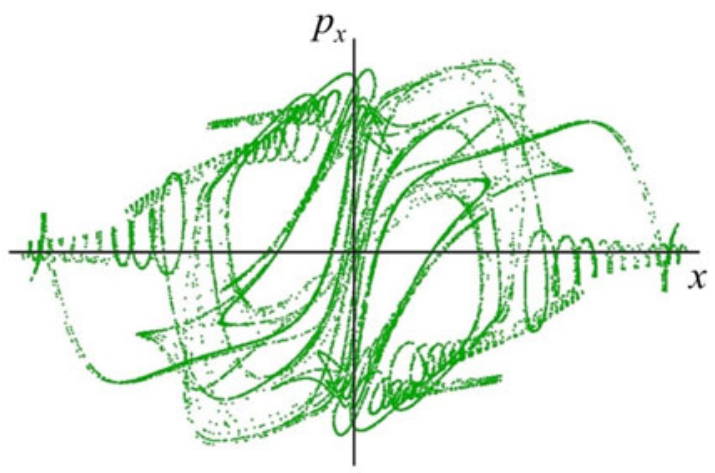

FIgURE 9. The Poincaré sections showing the particle positions in the phase plane $\left(x, p_{x}\right)$ at discrete times with the time step equal to the period of the driving force. The parameters are the same as in figure 8 for $x(0)=0.49$.

We also assume that the time average of the function $\xi(t)$ over the oscillation period $2 \pi / \omega$ is zero. Introducing the notation

$$
\langle x\rangle=\frac{\omega}{2 \pi} \int_{0}^{2 \pi / \omega} x(t) \mathrm{d} t,
$$

we obtain

$$
\langle\xi\rangle=\langle\dot{\xi}\rangle=\langle\ddot{\xi}\rangle=0 .
$$

Therefore, we have $\langle x\rangle=X(t)$, i.e. the function $X(t)$ describes the slow particle motion averaged over the fast oscillations, $\langle X\rangle \approx X(t)$.

Substituting (4.3) into the equation of particle motion (4.1) and expanding the functions $\kappa(x, t)$ and $F(x, t)$ in powers of $\xi$, i.e. writing $\kappa(x, t) \approx \kappa(X, t)+\xi \partial_{X} \kappa(X, t)$ and $F(x, t) \approx F(X, t)+\xi \partial_{X} F(X, t)$, we obtain

$$
\ddot{X}+\ddot{\xi}+\kappa \dot{X}+\kappa \dot{\xi}+\xi \dot{X} \partial_{X} \kappa+\xi \dot{\xi} \partial_{X} \kappa=F+\xi \partial_{X} F,
$$

where $\partial_{X}$ is the partial derivative with respect to the first argument of functions $\kappa(X, t)$ and $F(X, t)$. This equation contains slowly varying and fast oscillating terms, which apparently should be separately equal to each other. In the zeroth-order approximation with respect to small function $\xi$ and the time derivatives of the slow function $X$, we find the equation for the fast oscillating term

$$
\ddot{\xi}+\kappa \dot{\xi}=F .
$$

Here we neglect the terms proportional to $\xi$. The time derivatives $\ddot{\xi}$ and $\dot{\xi}$ are not small, being proportional to $\omega^{2}$ and $\omega$, respectively. They are assumed to be much greater than $\ddot{X}$ and $\dot{X}$. The friction coefficient $\kappa$ is not necessarily small.

Integration of (4.7), assuming $X$ to be constant, yields

$$
\begin{gathered}
\xi=\xi_{0}+\int_{0}^{t} \mathrm{~d} \tau\left[\mathrm{e}^{-K(X, \tau)} \dot{\xi}_{0}+\int_{0}^{\tau} \mathrm{e}^{K\left(X, \tau^{\prime}\right)-K(X, \tau)} F\left(X, \tau^{\prime}\right) \mathrm{d} \tau^{\prime}\right] \\
K(X, t)=\int_{0}^{t} \kappa(X, \tau) \mathrm{d} \tau .
\end{gathered}
$$


Assuming that $K(X, t)$ can be approximated by $K(X, t) \approx\langle\kappa\rangle t$, where $\langle\kappa\rangle$ is the timeaveraged friction coefficient, in the limit $t \gg 1 /\langle\kappa\rangle$ we obtain

$$
\begin{aligned}
\xi & =\frac{\left(\langle\kappa\rangle f_{1}-\omega f_{2}\right) \sin \omega t-\left(\langle\kappa\rangle f_{1}+\omega f_{2}\right) \cos \omega t}{\omega\left(\langle\kappa\rangle^{2}+\omega^{2}\right)} \\
& =-\frac{1}{\langle\kappa\rangle^{2}+\omega^{2}}\left(F+\frac{\langle\kappa\rangle}{\omega^{2}} \partial_{t} F\right)
\end{aligned}
$$

with $\partial_{t} F=\partial F(X, t) /\left.\partial t\right|_{X=\text { const }}$. Here we assume the initial condition $\xi_{0}=-\left(f_{2}+\right.$ $\left.\omega \dot{\xi}_{0}\right) /(\langle\kappa\rangle \omega)$.

Averaging (4.6) over time and taking into account that $\langle F(X, t)\rangle \approx 0$ for nearly constant $X(t)$, we obtain

$$
\ddot{X}+\left(\langle\kappa\rangle+\left\langle\xi \partial_{X} \kappa\right\rangle\right) \dot{X}=\left\langle\xi \partial_{X} F\right\rangle-\left\langle\xi \dot{\xi} \partial_{X} \kappa\right\rangle .
$$

Substituting expression (4.10) into the right-hand side of (4.11), for the first term we obtain

$$
\left\langle\xi \partial_{X} F\right\rangle=-\frac{\partial_{X}\left(f_{1}^{2}+f_{2}^{2}\right)}{4\left(\langle\kappa\rangle^{2}+\omega^{2}\right)}-\frac{\langle\kappa\rangle\left(f_{2} \partial_{X} f_{1}-f_{1} \partial_{X} f_{2}\right)}{2 \omega\left(\langle\kappa\rangle^{2}+\omega^{2}\right)} .
$$

The first term on the right-hand side of (4.12) is the well-known ponderomotive force (Landau \& Lifshitz 1976) where the friction effect is taken into account. The last term on the right-hand side, proportional to the friction coefficient, can change signs for $f_{2} \partial_{X} f_{1} \neq f_{1} \partial_{X} f_{2}$ depending on whether $f_{2} \partial_{X} f_{1}>f_{1} \partial_{X} f_{2}$ or $f_{2} \partial_{X} f_{1}<f_{1} \partial_{X} f_{2}$. It vanishes if $f_{2} \partial_{X} f_{1}=f_{1} \partial_{X} f_{2}, f_{1} \neq 0, f_{2}=0$ or $f_{2} \neq 0, f_{1}=0$.

The actual form of the last term on the right-hand side of (4.11) is determined by the specific dependence of the friction coefficient $\kappa$ on the driver force. As an example, we consider the case when this dependence is quadratic, i.e. $\kappa=v F^{2}$, with a constant $v$. Then we obtain

$$
-\left\langle\xi \dot{\xi} \partial_{X} \kappa\right\rangle=\frac{v\langle\kappa\rangle \partial_{X}\left(f_{1}^{2}+f_{2}^{2}\right)^{2}}{8\left(\langle\kappa\rangle^{2}+\omega^{2}\right)^{2}}+\frac{v\left(\langle\kappa\rangle^{2}-\omega^{2}\right)\left(f_{1}^{2}+f_{2}^{2}\right)\left(f_{2} \partial_{X} f_{1}-f_{1} \partial_{X} f_{2}\right)}{4 \omega\left(\langle\kappa\rangle^{2}+\omega^{2}\right)^{2}}
$$

and the time-averaged friction coefficient becomes $\langle\kappa\rangle=v\left(f_{1}^{2}+f_{2}^{2}\right) / 2$. In addition, $\left\langle\xi \partial_{X} \kappa\right\rangle=0$.

For the sake of simplicity we further assume that $f_{2}=0$ in expression (4.2) for the driver force. Then, (4.12) and (4.13) are simplified and we finally obtain the equation for the slowly varying function $X(t)$ :

$$
\ddot{X}+\frac{v f_{1}^{2}}{2} \dot{X}=-\frac{\partial_{X} f_{1}^{2}}{v^{2} f_{1}^{4}+4 \omega^{2}}+\frac{2 v^{2} \partial_{X} f_{1}^{6}}{3\left(v^{2} f_{1}^{4}+4 \omega^{2}\right)^{2}} .
$$

The first term on the right-hand side corresponds to the ponderomotive force, the last term is the drag force induced by the friction.

As we can see, the ponderomotive force (4.12) and the drag force due to the friction (4.13) have different signs in (4.14). If $|v|>2 \omega / f_{1}^{2}$, the drag force exceeds in magnitude the ponderomotive force.

Numerical integration of the equation of motion (4.1) with

$$
F(x, t)=f_{0} \exp \left(-\left(x / l_{0}\right)^{2}\right) \cos \omega t \quad \text { and } \quad \kappa=v F^{2}
$$



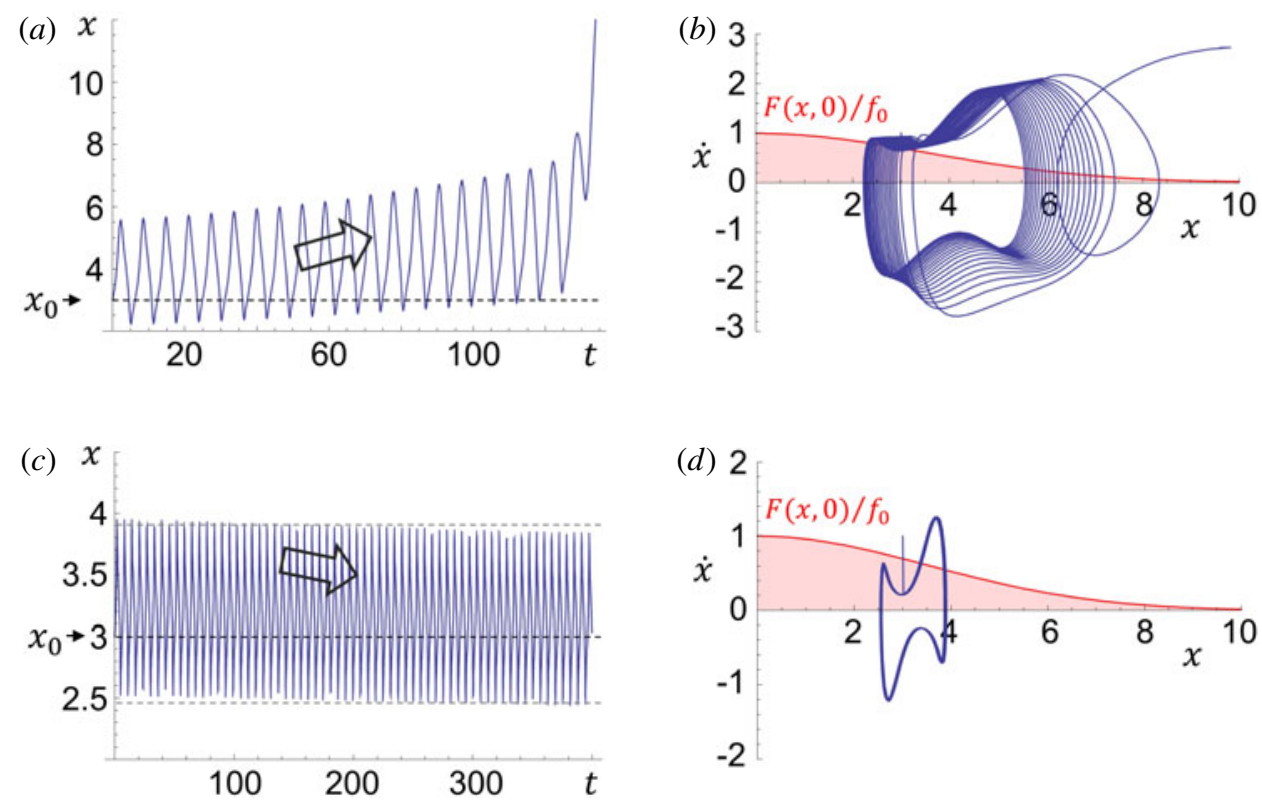

FIgURE 10. The solutions of (4.1) in the case of relatively weak driver force $(a, b)$, and for the case of relatively strong driver force $(c, d)$. $(a, c)$ Dependence of the particle coordinate on time. $(b, d)$ The particle trajectory in the phase plane $(x, \dot{x})$.

reveals the main features of the behaviour predicted within the framework of the simple model approximation. The solutions for the cases of relatively weak and relatively strong driver force are plotted in figure 10. The parameters are as follows. The driver frequency and the friction coefficient values are $\omega=1$ and $v=0.1$, respectively. The driver width equals $l_{0}=5$. The initial coordinate and velocity are $x_{0}=3$ and $\dot{x}_{0}=1$ in both cases. The driver amplitude is equal to $f_{0}=5 \sqrt{2 \omega / v}$ in the case of the weak driver and is equal to $f_{0}=15 \sqrt{2 \omega / v}$ in the case of the strong driver. As we see in figure $10(a, b)$, in the case of weak nonlinearity, the particle being pushed outwards by the ponderomotive force having performed several oscillations leaves the region where the driver force is localized. In contrast, in the limit of strong nonlinearity, the friction drag force prevents the particle from leaving the driver localization region resulting in its slow drift inwards (figure $10 c, d$ ).

On a trajectory corresponding to a quasi-periodic particle motion seen in figure 10 , the particle feels an almost constant driving force. This situation can be described in the approximation

$$
F(x, t)=f_{0} \cos \omega t, \quad \kappa=v f_{0}^{2} \cos ^{2} \omega t
$$

Substituting this driving force and friction coefficient into (4.1), we change variables to $(\tau, y(\tau)), t=\tau / \omega, x(t)=\left(f_{0} / \omega^{2}\right) y(\tau)$ and introduce the constant

$$
\sigma=v f_{0}^{2} / \omega
$$

Thus, we obtain

$$
y^{\prime \prime}(\tau)+\sigma \cos ^{2}(\tau) y^{\prime}(\tau)=\cos \tau
$$


Here a prime denotes a differentiation with respect to the variable $\tau$. Using (4.9) and the generating function for the modified Bessel functions of the first kind, $I_{k}$, one can cast the general solution to (4.18) in the form

$$
y^{\prime}(\tau)=\exp \left(-\frac{\sigma \tau}{2}-\frac{\sigma}{4} \sin (2 \tau)\right)\left[y^{\prime}(0)-Y_{L C}(0)\right]+Y_{L C}(\tau),
$$

where the function $Y_{L C}(\tau)$ is given by

$$
\begin{aligned}
Y_{L C}(\tau)= & \exp \left(-\frac{\sigma}{4} \sin (2 \tau)\right) \sum_{k=-\infty}^{\infty}(-\mathrm{i})^{k+1} I_{k}\left(\frac{\sigma}{4}\right) \\
& \times\left\{\frac{\exp [\mathrm{i}(2 k-1) \tau]}{4 k-2-\mathrm{i} \sigma}+\frac{\exp [\mathrm{i}(2 k+1) \tau]}{4 k+2-\mathrm{i} \sigma}\right\} .
\end{aligned}
$$

As one can see, any solution at $\tau \rightarrow \infty$ tends to the limit cycle described by the function $Y_{L C}$ and determined by the constant $y^{\prime}(0)=Y_{L C}(0)$.

The function describing the limit cycle, (4.20), can be represented as a Fourier series in terms of odd harmonics of the driving force frequency

$$
Y_{L C}(\tau)=\sum_{n=1}^{\infty}\left[\exp (\mathrm{i}(2 n-1) \tau) C_{n}(\sigma)+\exp (-\mathrm{i}(2 n-1) \tau) C_{1-n}(\sigma)\right]
$$

with

$$
C_{n}(\sigma)=\mathrm{i}^{n} \sum_{k=-\infty}^{\infty} \frac{(-1)^{k+1}}{4 k+2-\mathrm{i} \sigma}\left[I_{k}\left(\frac{\sigma}{4}\right)-\mathrm{i} I_{k+1}\left(\frac{\sigma}{4}\right)\right] I_{k+1-n}\left(\frac{\sigma}{4}\right) .
$$

This gives the spectrum of the limit cycle trajectory. For the particle velocity (corresponding to $y^{\prime}(\tau)$ ), the spectral density is $\left|2 C_{n}(\sigma)\right|^{2}$, figure 11 .

We believe that the approach formulated above towards dissipative stabilization of nonlinear dynamics systems will be useful for applications well beyond the framework of laser-matter interaction physics, remembering a saying of William Thomson (Lord Kelvin) 'I never satisfy myself until I can make a mechanical model of a thing' (see Thomson 1884).

\section{Regular and chaotic electron motion in three s- and p-polarized colliding laser pulses}

\subsection{EM field configuration}

Let us consider three $\mathrm{s}(\mathrm{p})$-polarized waves, in which the $z$-component of the electric (magnetic) field is given by

$$
\left(\begin{array}{l}
E_{z} \\
B_{z}
\end{array}\right)=-\frac{1}{\sqrt{3}}\left(\begin{array}{l}
E_{0} \\
B_{0}
\end{array}\right)\left\{\sin \left[\omega_{0}\left(t+\frac{y}{c}\right)\right]+2 \sin \left[\omega_{0}\left(t-\frac{y}{2 c}\right)\right] \cos \left(\omega_{0} \frac{\sqrt{3} x}{2 c}\right)\right\} .
$$

The $x$ and $y$ components of the magnetic (electric) field of the $\mathrm{s}(\mathrm{p})$-polarized wave are

$$
\left(\begin{array}{l}
B_{x} \\
E_{x}
\end{array}\right)=\frac{1}{\sqrt{3}}\left(\begin{array}{c}
E_{0} \\
-B_{0}
\end{array}\right)\left\{-\sin \left[\omega_{0}\left(t+\frac{y}{c}\right)\right]+\sin \left[\omega_{0}\left(t-\frac{y}{2 c}\right)\right] \cos \left(\omega_{0} \frac{\sqrt{3} x}{2 c}\right)\right\}
$$




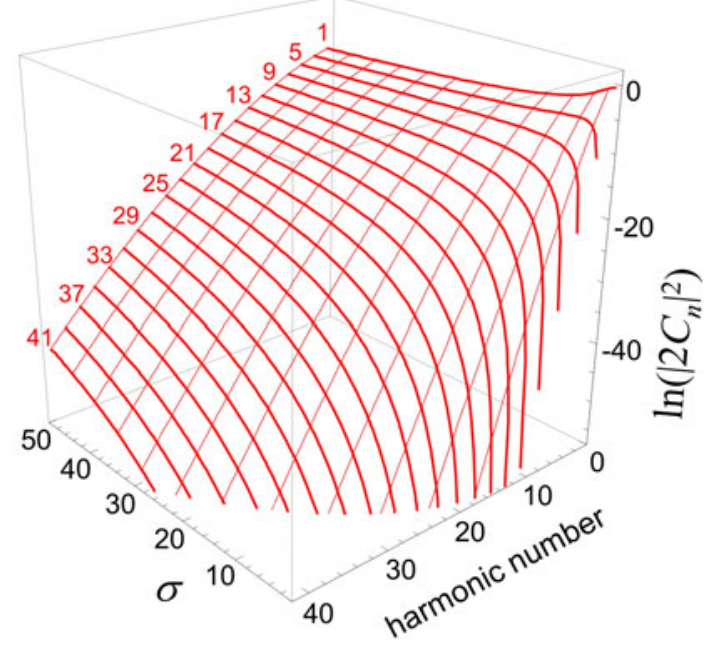

FIGURE 11. The spectral density of the particle velocity for several harmonics of the driving force frequency as a function of the friction parameter $\sigma$.

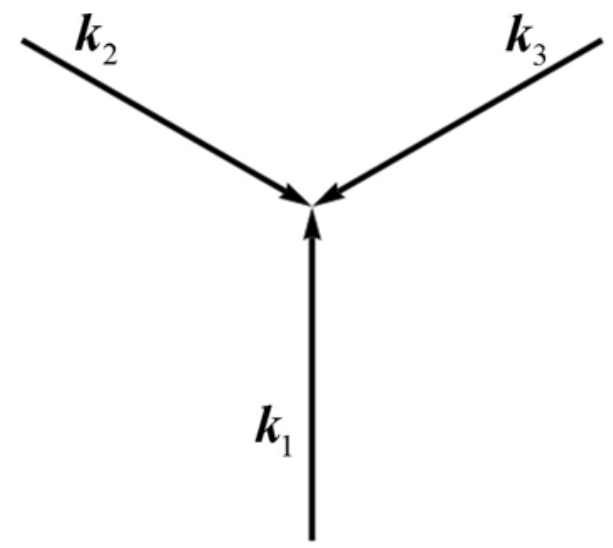

FIGURE 12. Wave vectors of three colliding waves.

and

$$
\left(\begin{array}{l}
B_{y} \\
E_{y}
\end{array}\right)=\frac{1}{\sqrt{3}}\left(\begin{array}{c}
E_{0} \\
-B_{0}
\end{array}\right) \cos \left[\omega_{0}\left(t-\frac{y}{2 c}\right)\right] \sin \left(\omega_{0} \frac{\sqrt{3} x}{2 c}\right) .
$$

The wave orientation is illustrated in figure 12. As an example in figure 13(a) we show the magnetic (electric) field $\boldsymbol{B}_{n}=B_{x} \boldsymbol{e}_{x}+B_{y} \boldsymbol{e}_{y}\left(\boldsymbol{E}_{n}=E_{x} \boldsymbol{e}_{x}+E_{y} \boldsymbol{e}_{y}\right)$ and in figure 13(b) the isocontours of the electric (magnetic) field $E_{z}\left(B_{z}\right)$ in the $(x, y)$ plane at time $t=\pi / 4$ for the case of three colliding s-polarized (p-polarized) EM waves.

\subsection{Electron interaction with three s-polarized EM waves}

\subsubsection{Particular solutions}

Due to the symmetry of the EM field given by (5.2), (5.3), there are particular solutions of the equations of motion, when the particle moves straight in the $(x, y)$ 

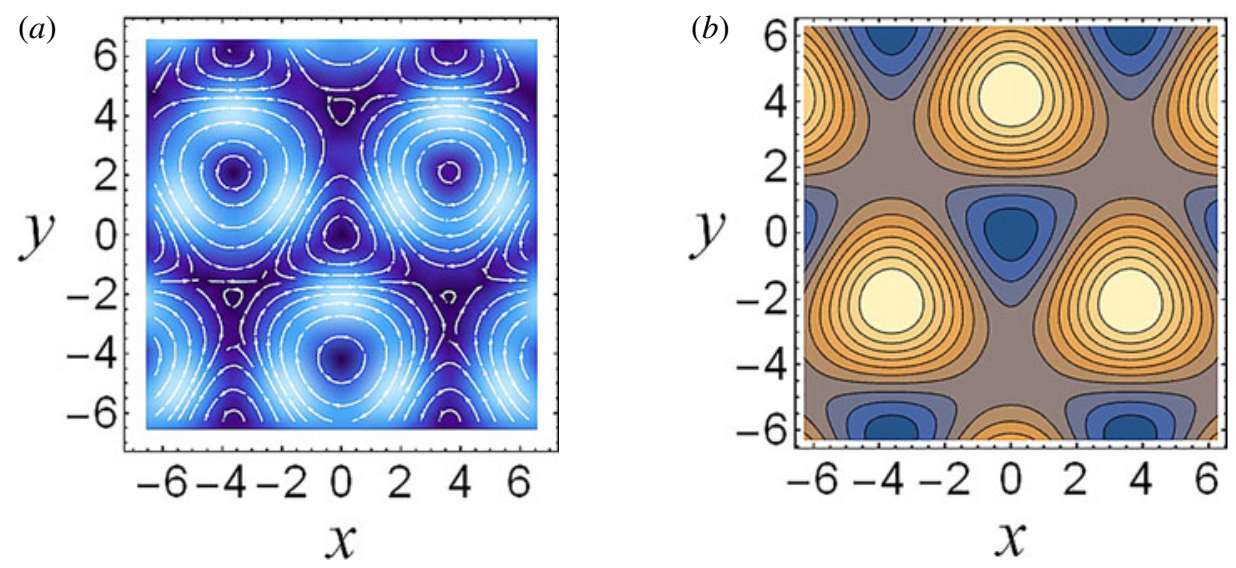

FIGURE 13. Three s-polarized (p-polarized) EM waves: (a) magnetic (electric) field; (b) isocontours of the electric (magnetic) field in the $(x, y)$ plane at time $t=\pi / 4$.

plane along the direction of one of the waves propagation. If we let $x=0$ in (5.2), (5.3), the electromagnetic field formally corresponds to a superposition of two EM waves one of which propagates with the velocity equal to $-c$ and another has the velocity $2 c$.

The integration of the equations of electron motion yields the particle trajectories presented in figure 14 for initial conditions: $x(0)=0, y(0)=0.05, z(0)=0, p_{x}(0)=0$, $p_{y}(0)=0, p_{z}(0)=0$. The normalized electromagnetic field amplitude is $a_{0}=436$ (each of the colliding waves has the amplitude equal to $a_{0} / 3$ ), the dissipation parameter is $\varepsilon_{\text {rad }}=1.2 \times 10^{-8}$ and the normalized critical QED field is $a_{S}=4 \times 10^{5}$. The electron trajectory in the $(y, z)$ plane plotted in figure $14(a)$ and the trajectory in the phase $\left(y, p_{z}\right)$ plane shown in figure $14(b)$ look similar to the trajectories presented in figure $2(a, b)$. The particle is trapped for a finite time within the EM field period performing relatively small-scale oscillations. Then, after some time it over-leaps to the next EM field period. This is also clearly seen in figure 14(c), where its $y$-coordinate is plotted versus time. From the Poincaré sections in figure $14(d)$, which show the particle positions in the phase plane $\left(p_{z}, p_{y}\right)$ at discrete times with the time step equal to the period of the driving force, we may see that this process is stochastic. The particle over-leaping from one field period to another with small-scale oscillations in between (see figures 2, 3 and 14) may be interpreted in terms of Lévy flights (Lévy 1954; Metzler \& Klafter 2000; Zaslavsky 2002; Metzler et al. 2007).

The case of high laser amplitude is presented in figure 15 for initial conditions: $x(0)=0, y(0)=-0.0001, z(0)=0, p_{x}(0)=0, p_{y}(0)=0, p_{z}(0)=0$. The normalized electromagnetic field amplitude is $a_{0}=4700$ (each of the colliding waves has the amplitude equal to $a_{0} / 3$ ), the dissipation parameter is $\varepsilon_{\text {rad }}=1.2 \times 10^{-9}$ and the normalized critical QED field is $a_{S}=4 \times 10^{6}$. The electron trajectory in the $(y, z)$ plane plotted in figure $15(a)$ and the trajectory in the phase $\left(y, p_{y}\right)$ plane shown in figure $15(b)$ clearly demonstrate the particle trapping in the limit circle after an initial phase corresponding to the particle motion in the vicinity of the electric field node, $y=0$. Since the motion here is unstable, the particle leaves this region. This is also distinctly seen in figure $15(d)$ showing the electron trajectory in the $\left(p_{y}, p_{z}\right)$ plane. In the plane $(y, z)$ (figure $15 a)$ as we see, when the particle moves along the 
(a) $z$

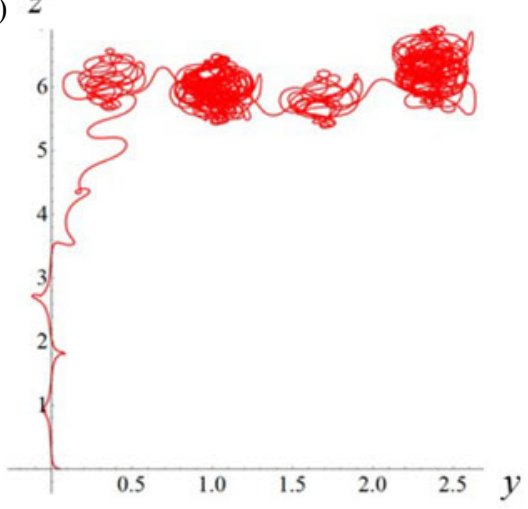

(c) $y$

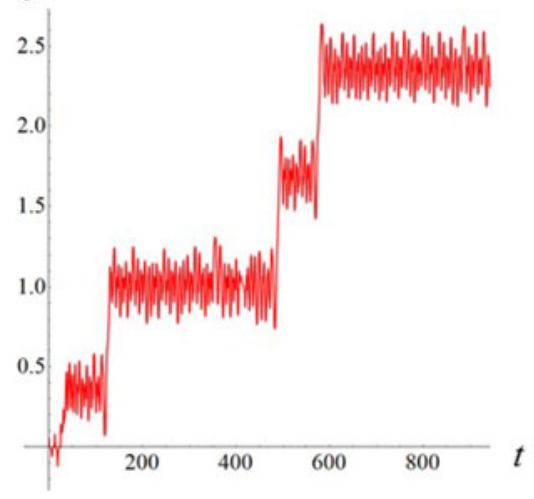

(b)

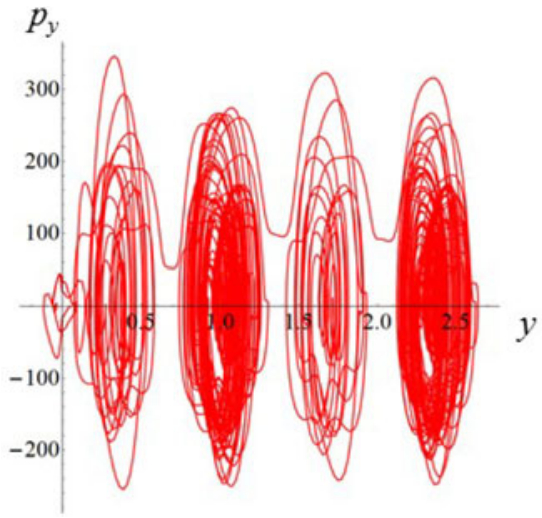

(d)

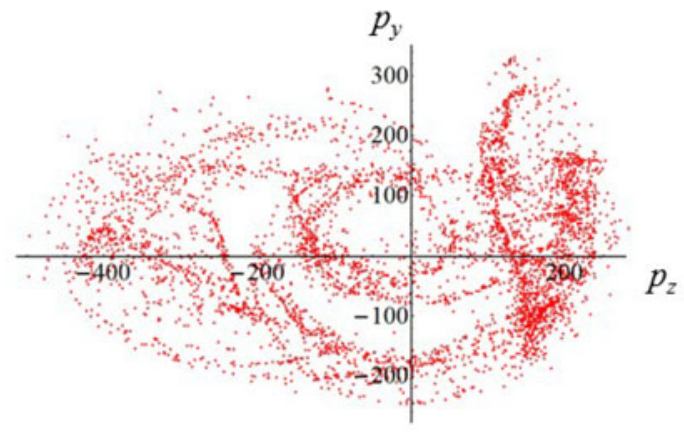

FIGURE 14. (a) Electron trajectory in the $(y, z)$ plane for initial conditions: $x(0)=0$, $y(0)=0.05, z(0)=0, p_{x}(0)=0, p_{y}(0)=0, p_{z}(0)=0$. (b) Trajectory in the phase $\left(y, p_{z}\right)$ plane; $(c)$ electron $y$-coordinate versus time; $(d)$ the Poincare sections showing the particle positions in the phase plane $\left(p_{z}, p_{y}\right)$ at discrete times with the time step equal to the period of the driving force. The electromagnetic field amplitude is $a_{0}=436$, the dissipation parameter is $\varepsilon_{\text {rad }}=1.2 \times 10^{-8}$ and the normalized critical QED field is $a_{S}=4 \times 10^{5}$. The coordinates, time and momentum are measured in $2 \pi c / \omega, 2 \pi / \omega$ and $m_{e} c$ units.

limit circle, its trajectory has the 'figure eight' form. It performs regular oscillations (see figure $15 c$, where the particle coordinate $y$ is plotted versus time) with the double frequency for oscillations along the $y$ axis compared with the frequency of oscillation along the $z$ axis.

5.2.2. Random walk and regular patterns of the particle trajectories in the field of three 3 s-polarized EM waves

Results of the integration of the motion equations for the electron interacting with three 3 s-polarized EM waves in the limit of relatively low radiation intensity are presented in figure 16. Figure 16(a) shows 8 electron trajectories in the $(x, y)$ plane for initial conditions: $x(0)$ and $y(0)$ are in the vicinity of the coordinate origin, and $z(0)=0, p_{x}(0)=0, p_{y}(0)=0, p_{z}(0)=0$. In figure $16(b)$ we plot a close up of the trajectories in the vicinity of the coordinate origin superimposed with 
(a)

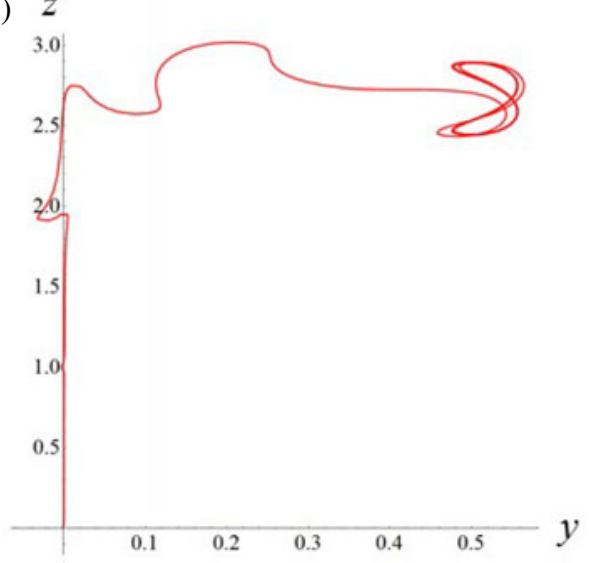

(c)

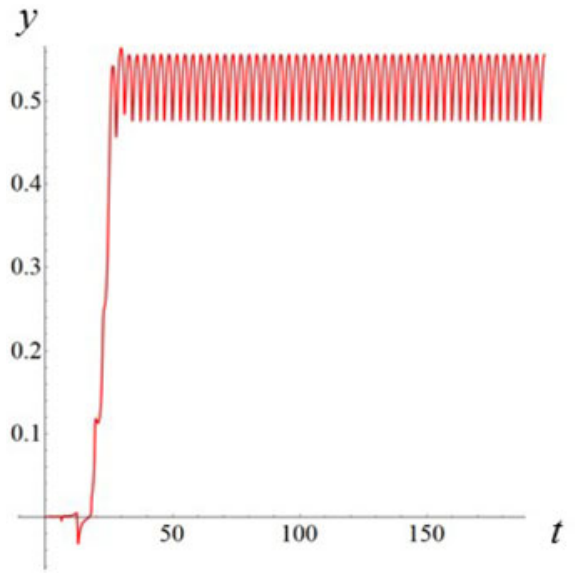

(b)

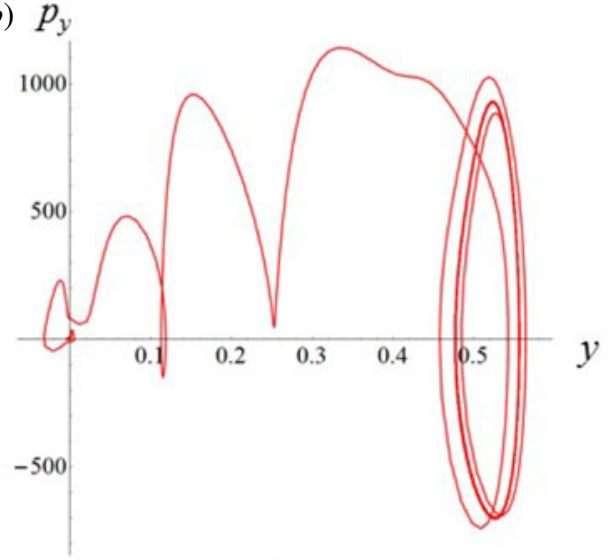

(d)

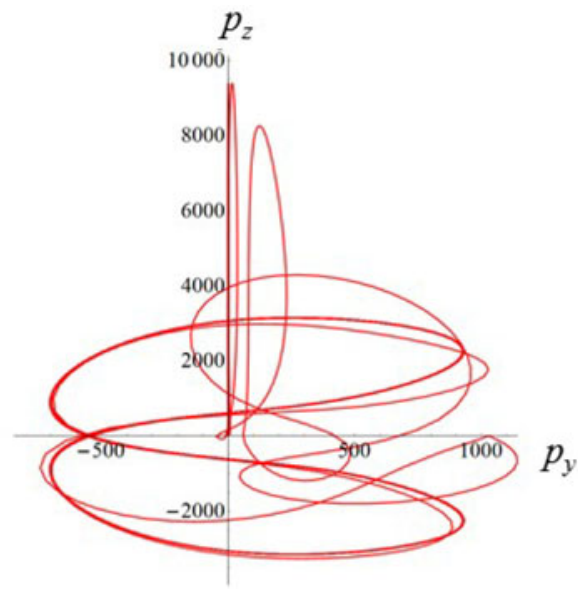

FIGURE 15. (a) Electron trajectory in the $(y, z)$ plane for initial conditions: $x(0)=0$, $y(0)=-0.0001, z(0)=0, p_{x}(0)=0, p_{y}(0)=0, p_{z}(0)=0 .(b)$ Trajectory in the phase plane $\left(y, p_{y}\right) ;(c)$ electron $y$-coordinate versus time; $(d)$ electron trajectory in the $\left(p_{y}, p_{z}\right)$ plane. The electromagnetic field amplitude is $a_{0}=4700$, the dissipation parameter is $\varepsilon_{\text {rad }}=$ $1.2 \times 10^{-9}$ and the normalized critical QED field is $a_{S}=4 \times 10^{6}$. The coordinates, time and momentum are measured in $2 \pi c / \omega, 2 \pi / \omega$ and $m_{e} c$ units.

the isocontours of the electromagnetic potential averaged over a half-period of the field oscillations. It is proportional to the ponderomotive potential in the high field amplitude limit, $a_{0} \gg 1$. As we see the typical trajectories are comprised of long range Lévy-flight-like excursions and short range rambling motion, which changes the direction of succeeding flight. The combination of the long range excursions and short range rambling is also seen in the dependence of the electron $y$ coordinate on time in figure $16(d)$. The corresponding particle trajectory in the $p_{x}, p_{y}, p_{z}$ momentum space for $x(0)=-0.125$ and $y(0)=0.125$ is presented in figure $16(c)$. What is remarkable is that during the Lévy-like flights the electron moves almost along one of the three waves propagation direction (compare figures 12 and 16a). This stage of the particle motion can be described by the particular solution analysed above and illustrated in figure 14. 
(a)

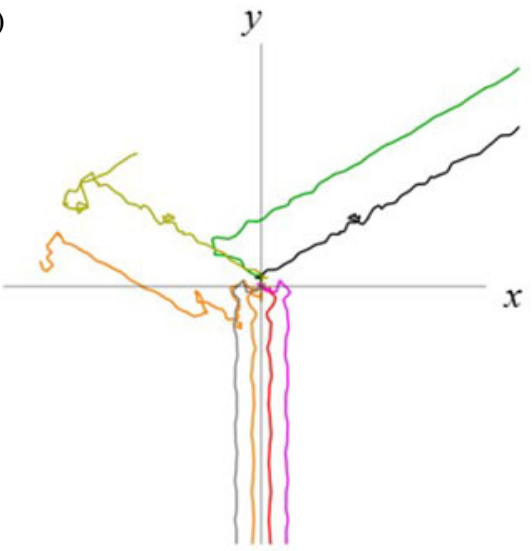

(c)

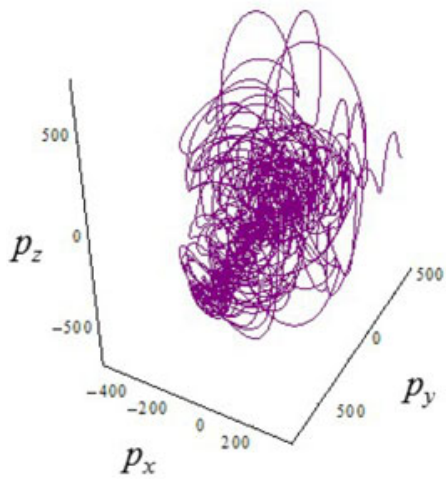

(b)

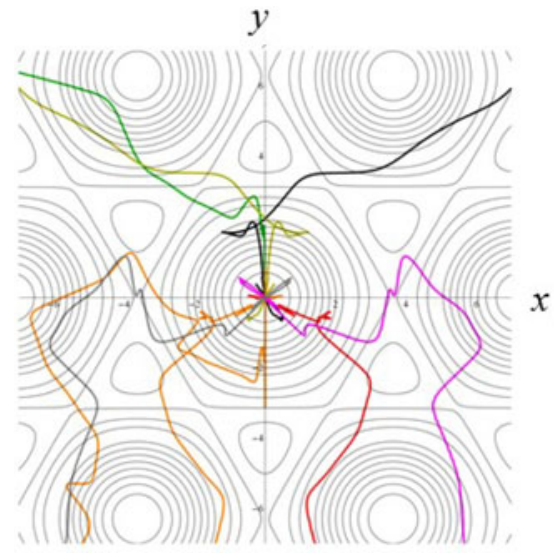

(d)

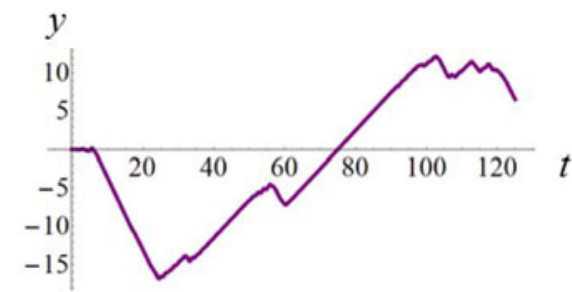

FIGURE 16. (a) Eight electron trajectories in the $(x, y)$ plane for initial conditions: $x(0)$ and $y(0)$ are in the vicinity of the coordinate origin, and $z(0)=0, p_{x}(0)=0$, $p_{y}(0)=0, p_{z}(0)=0$. (b) Close up of the trajectories in the vicinity of the coordinate origin. (c) Electron trajectory in the $p_{x}, p_{y}, p_{z}$ space for $x(0)=-0.125$ and $y(0)=$ 0.125. (d) Electron $y$ coordinate versus time for $x(0)=-0.125$ and $y(0)=0.125$. The electromagnetic field amplitude is $a_{0}=756$, the dissipation parameter is $\varepsilon_{\text {rad }}=1.2 \times$ $10^{-8}$ and the normalized critical QED field is $a_{S}=4 \times 10^{5}$. The coordinates, time and momentum are measured in $2 \pi c / \omega, 2 \pi / \omega$ and $m_{e} c$ units.

Qualitatively different patterns formed by the trajectories of particles interacting with the field of three 3 s-polarized EM waves are observed in the high intensity and low frequency limit. These patterns are shown in figure $17(a)$ and in figure $17(b)$ presenting a close up of the trajectories in the vicinity of the coordinate origin, where the trajectories in the $(x, y)$ plane of an electron ensemble make a tracery strikingly remeniscent of parquetry or window frost. Either an individual trajectory or the ensemble appears to be confined in the lower measure sub-domain periodic in the $x$ and $y$ directions. In figure $17(c)$ the electron trajectory in the $p_{x}, p_{y}, p_{z}$ momentum space for $x(0)=-0.125$ and $y(0)=0.125$ demonstrates that the particle energy remains finite. The electron $y$ coordinate dependence on time for $x(0)=-0.001$ and $y(0)=-0.001$ plotted in figure $17(c)$ shows that the particle motion is comprised of relatively long over-leaps interlaced with small-scale oscillations. In figure 17(e) we present the corresponding Poincare sections, i.e. we plot the particle positions in the phase plane $\left(p_{x}, p_{y}\right)$ at discrete times with the time step equal to the period 
(a)

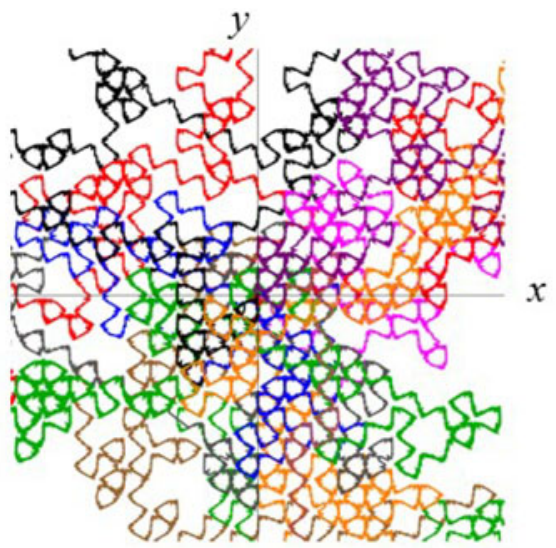

(c)

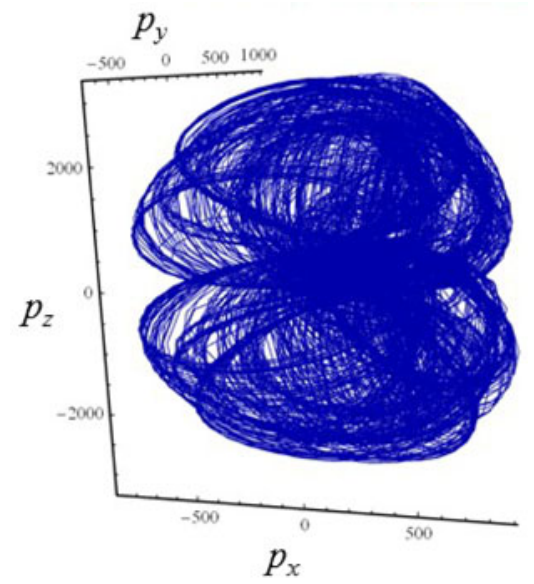

(b)

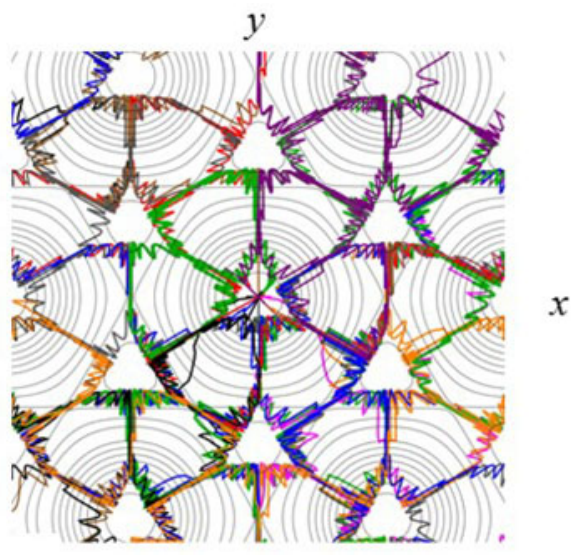

(d) $y$

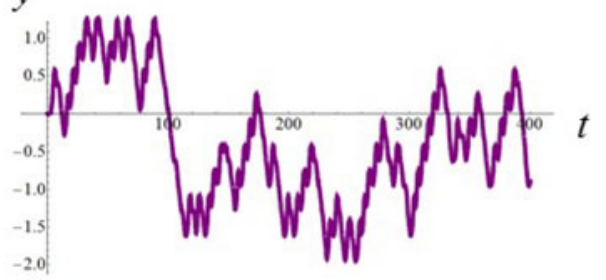

(e)

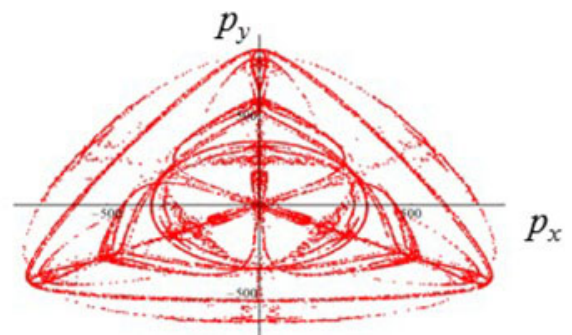

FIGURE 17. (a) Ensemble of electron trajectories in the $(x, y)$ plane for initial conditions: $x(0)$ and $y(0)$ are in the vicinity of the coordinate origin, and $z(0)=0, p_{x}(0)=0$, $p_{y}(0)=0, p_{z}(0)=0$. (b) Close up of the trajectories in the vicinity of the coordinate origin. (c) Electron trajectory in the $p_{x}, p_{y}, p_{z}$ space for $x(0)=-0.125$ and $y(0)=0.125$. (d) Electron $y$ coordinate versus time for $x(0)=-0.001$ and $y(0)=-0.001$. (e) The Poincare sections: the particle positions in the phase plane $\left(p_{x}, p_{y}\right)$ at discrete times with the time step equal to the period of the driving force. The electromagnetic field amplitude is $a_{0}=4764$, the dissipation parameter is $\varepsilon_{\text {rad }}=6 \times 10^{-9}$ and the normalized critical QED field is $a_{S}=8 \times 10^{5}$. The coordinates, time and momentum are measured in $2 \pi c / \omega, 2 \pi / \omega$ and $m_{e} c$ units. The integration time equals $200 \times 2 \pi / \omega$.

of the driving force. The Poincare sections, in this case, indicate that the particle motion is pretty regular. Here the parameters of the EM field and of the electrons are as follows. The electromagnetic field amplitude is $a_{0}=4764$ (the amplitude of each colliding wave is equal to 1588), the dissipation parameter is $\varepsilon_{\text {rad }}=6 \times 10^{-9}$ and the normalized critical QED field is $a_{S}=8 \times 10^{5}$, which corresponds to the wave frequency a factor two smaller than in the case shown in figure 16. The integration time equals $200 \times 2 \pi / \omega$. 


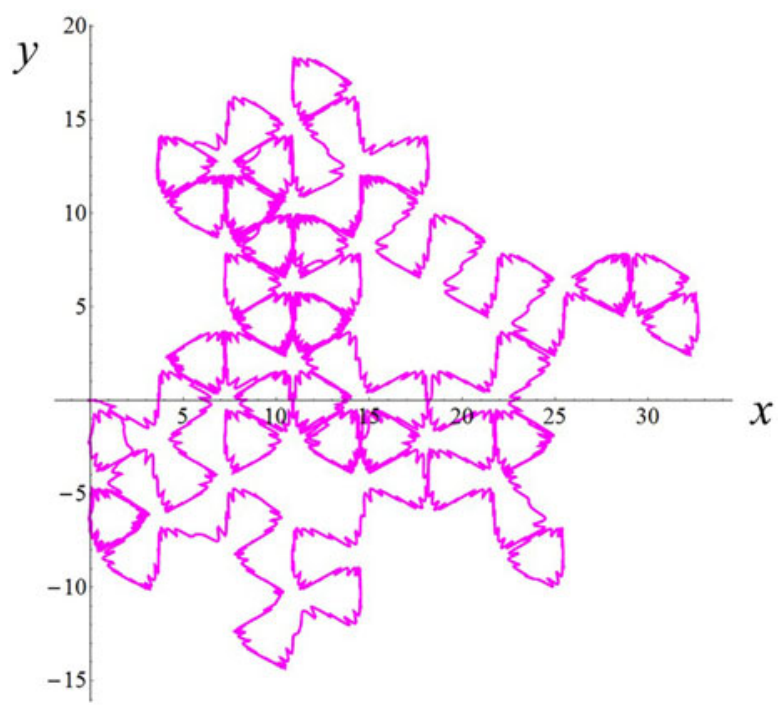

FIGURE 18. Trajectory of the electron migrating over a long time in the $(x, y)$ plane.

\subsubsection{Ergodization or not?}

The attractor trajectory pattern in figure $17(a, b)$ is made by an ensemble of electrons. The single electron trajectory shown in figure 18 demonstrates that, having been moving for a long enough time, it could cover the whole attractor. In view of this, there are two questions. The first one being whether there is an analogy of the ergodic hypothesis saying that over long periods of time, the time spent in some region of the attractor is proportional to the attractor measure? The second one being whether there is an analogy of the Poincaré recurrence theorem (for details see Arnol'd 1989) saying that the particle, after a sufficiently long but finite time, returns to a point very close to the initial point? A similar question occurs in the case of the particle random walk regarding whether the results of the well-known random walk theory (Kac 1961) can be used in our case. In both the regular and stochastic regimes the methods developed in turbulence theory (see Mathur et al. 2007; Peacock \& Haller 2013) can be used for analysing and identifying the attractors and repelling trajectories.

\subsection{Electron interaction with three p-polarized EM waves}

In the case of three p-polarized EM waves the EM configuration is described by (5.1)-(5.3). As in the s-polarization case, in the limit of relatively low EM wave intensity the electron performs the random walk motion comprised of short scalelength oscillations interleaved by long scale-length Lévy-like flights. An example of such the trajectory is shown in figure 19(a) for the EM field amplitude of $a_{0}=4764$, the dissipation parameter of $\varepsilon_{\text {rad }}=6 \times 10^{-9}$ and the normalized critical QED field of $a_{S}=8 \times 10^{5}$. For the high intensity EM wave case the electrons migrate along the paths confined in narrow valleys, as can be seen in figure $19(b)$, where the ensemble of the electron trajectories is plotted for the EM field amplitude of $7.2 \times 10^{3}$, the dissipation parameter of $\varepsilon_{\text {rad }}=1.2 \times 10^{-8}$ and the normalized critical QED field of $a_{S}=4.1 \times 10^{5}$. 
(a)

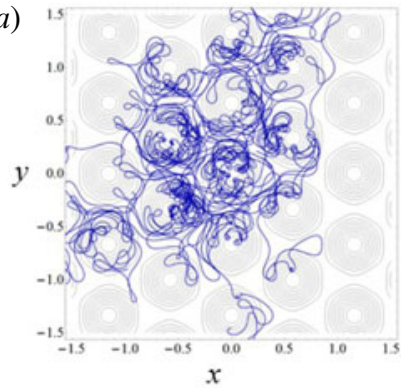

(d)

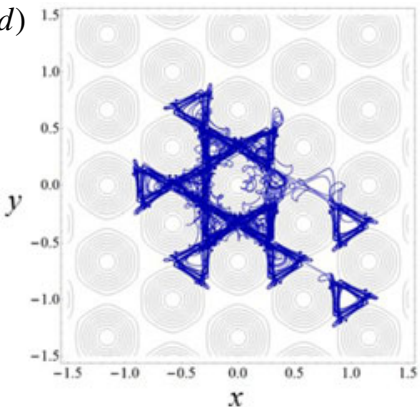

(b)

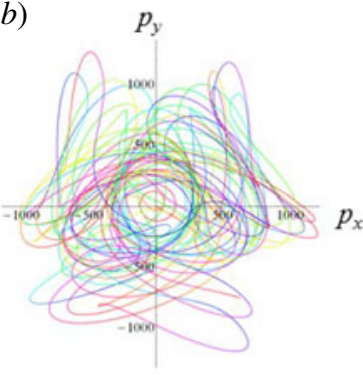

(e)

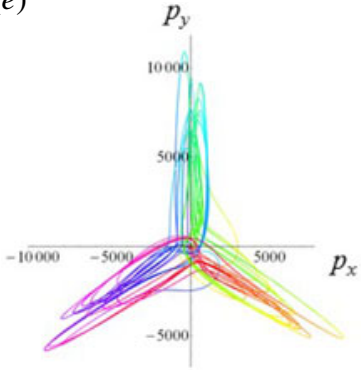

(c)

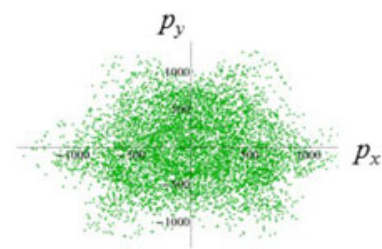

(f)

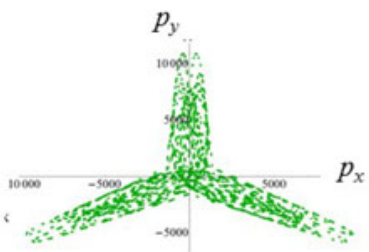

FIGURE 19. (a) Ensemble of electron trajectories in the $(x, y)$ plane for initial conditions: $x(0)$ and $y(0)$ are in the vicinity of the coordinate origin, and $z(0)=0, p_{x}(0)=0, p_{y}(0)=0$, $p_{z}(0)=0$. (b) Electron trajectories in the $\left(p_{x}, p_{y}\right)$ plane. $(c)$ The Poincare sections: the particle positions in the phase plane $\left(p_{x}, p_{y}\right)$ at discrete times with the time step equal to the period of the driving force. The electromagnetic field amplitude is $a_{0}=1383$, the dissipation parameter is $\varepsilon_{\text {rad }}=1.2 \times 10^{-6}$ and the normalized critical QED field is $a_{S}=$ $4.1 \times 10^{5}$. (d) Ensemble of electron trajectories in the $(x, y)$ plane for initial conditions: $x(0)$ and $y(0)$ are in the vicinity of the coordinate origin, and $z(0)=0, p_{x}(0)=0, p_{y}(0)=0$, $p_{z}(0)=0$. $(e)$ Electron trajectories in the $\left(p_{x}, p_{y}\right)$ plane. $(f)$ The Poincare sections: the particle positions in the phase plane $\left(p_{x}, p_{y}\right)$ at discrete times with the time step equal to the period of the driving force. The electromagnetic field amplitude is $a_{0}=7.2 \times 10^{3}$, the dissipation parameter is $\varepsilon_{\text {rad }}=1.2 \times 10^{-8}$ and the normalized critical QED field is $a_{S}=4.1 \times 10^{5}$.

\section{Electron dynamics in four s- and p-polarized colliding EM pulses}

The orientation of four colliding waves is illustrated in figure 20. Figure 21(a) shows magnetic (electric) field and $(b)$ isocontours of the electric (magnetic) field in the $(x, y)$ plane at time $t=\pi / 4$ of four s-polarized (p-polarized) colliding EM waves.

\subsection{S-polarized 4 colliding EM waves}

\subsubsection{EM field configuration}

In the EM configuration of four colliding $\mathrm{s}(\mathrm{p})$-polarized waves the $z$-components of the electric (magnetic) field can be written

$$
\left(\begin{array}{l}
E_{z} \\
B_{z}
\end{array}\right)=\left(\begin{array}{l}
E_{0} \\
B_{0}
\end{array}\right) 2 \sin \left(\omega_{0} t\right)\left[\cos \left(\omega_{0} \frac{x}{c}\right)+\cos \left(\omega_{0} \frac{y}{c}\right)\right] .
$$




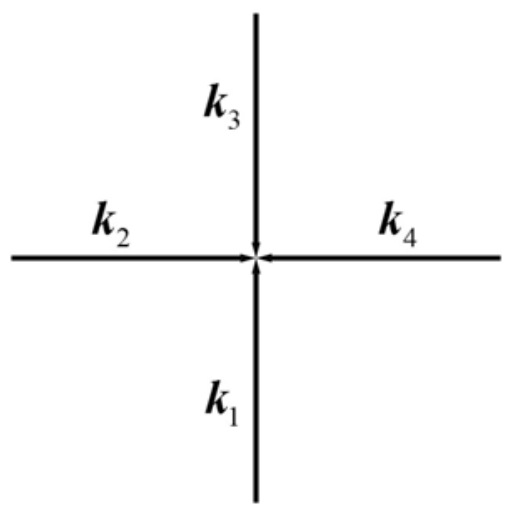

FIGURE 20. Wave vectors of four colliding EM waves.
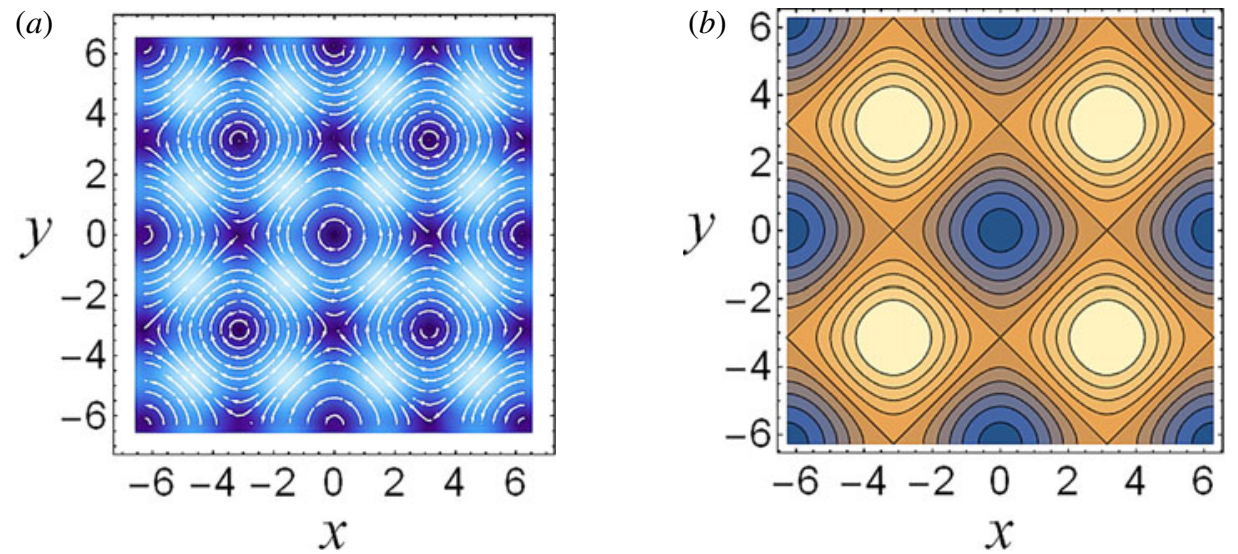

FIGURE 21. Four s-polarized (p-polarized) EM waves: (a) magnetic (electric) field;

(b) isocontours of the electric (magnetic) field in the $(x, y)$ plane at time $t=\pi / 4$.

The $x$ and $y$ components of the magnetic (electric) field of the four colliding $\mathrm{s}(\mathrm{p})$ polarized waves are given by

$$
\left(\begin{array}{c}
B_{x} \\
E_{x}
\end{array}\right)=\left(\begin{array}{c}
-E_{0} \\
B_{0}
\end{array}\right) 2 \cos \left(\omega_{0} t\right) \sin \left(\omega_{0} \frac{y}{c}\right)
$$

and

$$
\left(\begin{array}{c}
B_{y} \\
E_{y}
\end{array}\right)=\left(\begin{array}{c}
E_{0} \\
-B_{0}
\end{array}\right) 2 \cos \left(\omega_{0} t\right) \sin \left(\omega_{0} \frac{x}{c}\right),
$$

respectively.

\subsubsection{Particular solutions}

As in the above considered case of three s-polarized EM waves the equations of electron motion admit particular solutions, in the first of which the particle moves either along one of the axis, i.e. $x=n c \pi / \omega$ or $y=n c \pi / \omega$ with $n=0, \pm 1, \pm 2, \ldots$, and in the second it moves along straight lines $x= \pm y+n c \pi / \omega$. 
(a)

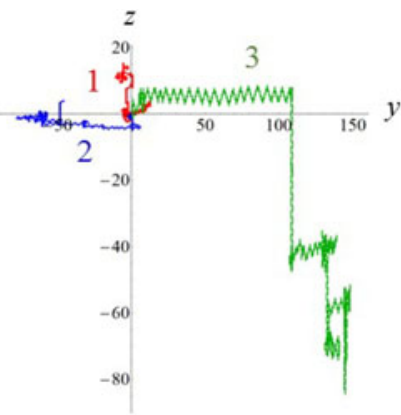

(d) 2

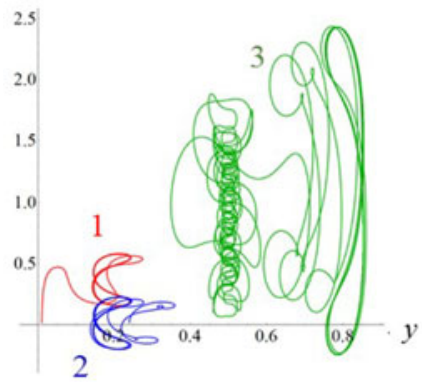

(b)

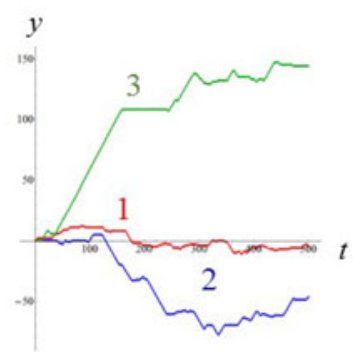

(e)

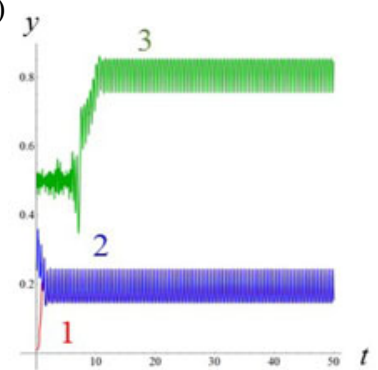

(c)

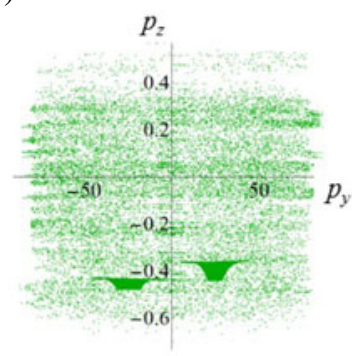

(f)

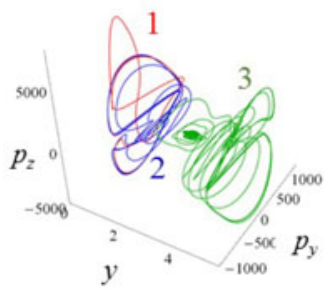

FIGURE 22. Electron trajectories in the case of the first type particular solution corresponding to the motion along the $y$ axis $($ at $x=0)$ in the field of four colliding EM waves for $\varepsilon_{\text {rad }}=1.2 \times 10^{-9}, a_{S}=4 \times 10^{6}, a=94, \omega=0.1$ for initial conditions: $y(0)=0.01, z(0)=0, p_{y}(0)=0, p_{z}(0)=0(\mathrm{red},-1) ; y(0)=0.23, z(0)=0, p_{y}(0)=0$, $p_{z}(0)=0$ (blue, -2$) ; y(0)=0.45, z(0)=0, p_{x}(0)=0, p_{z}(0)=0$ (green, -3 ). (a) Trajectories in the $(y, z)$ plane. $(b)$ Dependences of the $y$ coordinates on time. (c) The Poincare sections: the particle positions in the phase plane $\left(p_{y}, p_{z}\right)$ at discrete times with the time step equal to the period of the driving force. For lower frequency, $\omega=0.02$, when $\varepsilon_{\text {rad }}=2.4 \times 10^{-10}, a_{S}=2 \times 10^{7}, a_{0}=8 \times 10^{3}$, they are shown $(d)$ trajectories in the $(y, z)$ plane, $(e)$ dependences of the $y$ coordinates on time and $(f)$ trajectories in the $\left(y, p_{y}, p_{z}\right)$ space for the same initial conditions as in the panels $(a-c)$.

First type solution. For the first class of particular solutions with $x=n c \pi / \omega$ (without loss of generality we may take $n=0$, i.e. consider $x=0$ ), formally the particle moves in a superposition of the fields of two counter-propagating s-polarized EM waves and a homogeneous oscillating electric field directed along the $z$ axis. As in the above considered cases of two and three colliding EM waves, in the limit of weak nonlinearity and dissipation $\left(\varepsilon_{\text {rad }}=1.2 \times 10^{-9}, a_{S}=4 \times 10^{6}, a=94, \omega=0.1\right.$, for initial conditions: $y(0)=0.01, z(0)=0, p_{x}(0)=0, p_{z}(0)=0(\mathrm{red},-1) ; y(0)=0.23$, $z(0)=0, p_{x}(0)=0, p_{z}(0)=0$ (blue, -2$) ; y(0)=0.45, z(0)=0, p_{x}(0)=0, p_{z}(0)=0$ (green, -3)) the particle motion can be described as a random walk, for which the trajectories consist of the relatively small amplitude fast oscillating parts and of the long scale-length Lévy flights (see figure 22a,b). The Poincare sections, the particle positions in the phase plane $\left(p_{y}, p_{z}\right)$ at discrete times with the time step equal to the period of the driving force, presented in figure $22(c)$, show that the electron motion is stochastic.

In the case of lower frequency, $\omega=0.02$, and higher dimensionless EM field amplitude $a=8 \times 10^{3}$, when $\varepsilon_{\text {rad }}=2.4 \times 10^{-10}, a_{S}=2 \times 10^{7}, a_{0}=8 \times 10^{3}, \omega=0.02$, 
(a)

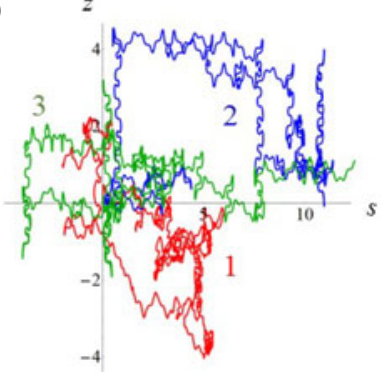

(d)

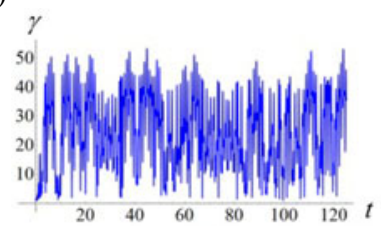

(b)

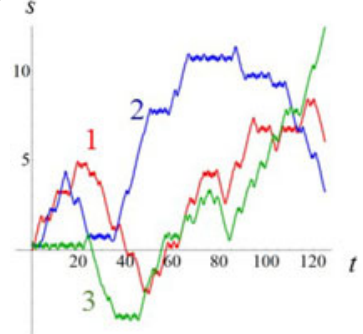

$(e)$

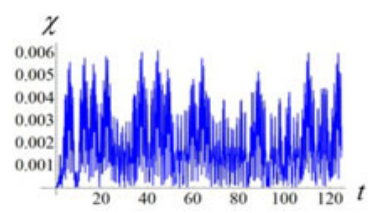

(c)

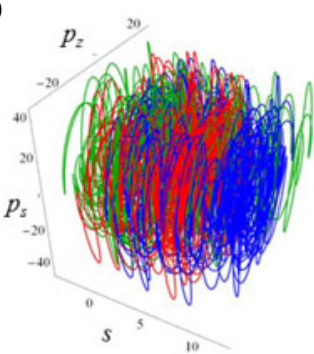

$(f)$

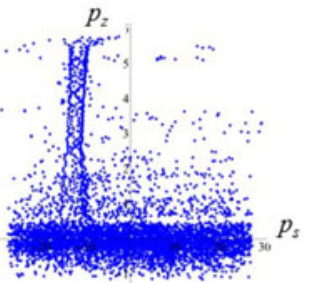

FIGURE 23. Electron trajectories in the case of the second type particular solution corresponding to the motion along the $x=y$ direction in the field of four colliding EM waves for $\varepsilon_{\text {rad }}=1.2 \times 10^{-8}, a_{S}=4 \times 10^{5}, a_{0}=44, \omega=1$ for initial conditions: $x(0)=0.01$, $z(0)=0, p_{x}(0)=0, p_{z}(0)=0(\mathrm{red},-1) ; x(0)=0.23, z(0)=0, p_{x}(0)=0, p_{z}(0)=0$ (blue, -2$) ; x(0)=0.45, z(0)=0, p_{x}(0)=0, p_{z}(0)=0$ (green, -3 ). (a) Trajectories in the $(s, z)$ plane. (b) Dependences of the $s$ coordinates on time. (c) Trajectories in the $\left(s, p_{s}, p_{z}\right)$ space. $(d)$ The particle Lorentz factor $\gamma$ versus time. $(e)$ Parameter $\chi$ versus time. $(f)$ The Poincare sections: the particle positions in the phase plane $\left(p_{s}, p_{z}\right)$ at discrete times with the time step equal to the period of the driving force.

the electron trajectories in the the $(y, z)$ plane (see figure $22 d$ ) show that the particles are trapped within narrow regions moving along regular limit circles (figure 22e). The attractor geometry is distinctly seen in figure $22(f)$, where the trajectories in the $\left(y, p_{y}, p_{z}\right)$ space are presented. As well seen, after a relatively short initial time interval the particles are trapped into stable limit circles performing periodic motion. We note that for the parameters chosen although the particle energy is ultrarelativistic the value of $\chi_{e}$ remains below unity, i.e. the QED effect of the recoil is not significant.

Second type solution. The particle behaviour under the conditions corresponding to the second class of particular solutions of the equations of motion $(x=y)$ is illustrated in figures 23 and 24. Here the coordinate $s(t)$ is equal to $s=x=y$.

In figure 23 we present electron trajectories in the case corresponding to the motion along the $x=y$ direction in the field of four colliding EM waves for $\varepsilon_{\text {rad }}=1.2 \times 10^{-8}$, $a_{S}=4 \times 10^{5}, a_{0}=44, \omega=1$ for initial conditions: $x(0)=0.01, z(0)=0, p_{x}(0)=0$, $p_{z}(0)=0(\mathrm{red},-1) ; x(0)=0.23, z(0)=0, p_{x}(0)=0, p_{z}(0)=0$ (blue, -2$) ; x(0)=0.45$, $z(0)=0, p_{x}(0)=0, p_{z}(0)=0$ (green, -3$)$. Figure 23(a) shows electron trajectories in the $(s, z)$ plane, which demonstrate random walks with intermittent short scale-length oscillations and long range Lévy flights. The same behaviour is distinctly seen in figure 23(b) with three dependences of the $s$ coordinates on time. Stochastic character of the particle motion is demonstrated in figure $23(b, f)$ by the behaviour of trajectories in the $\left(s, p_{s}, p_{z}\right)$ space and by the particle positions in the phase plane 
(a)

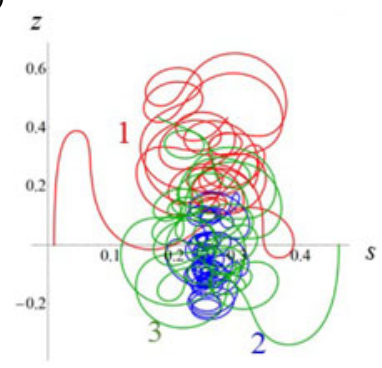

$(d)$

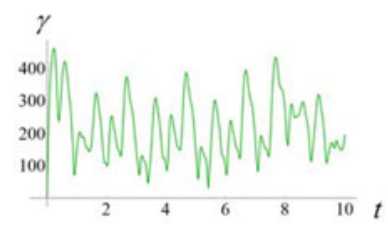

(b)

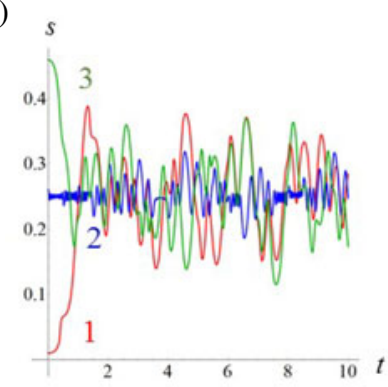

(e)

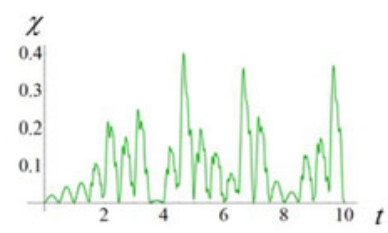

$(c)$

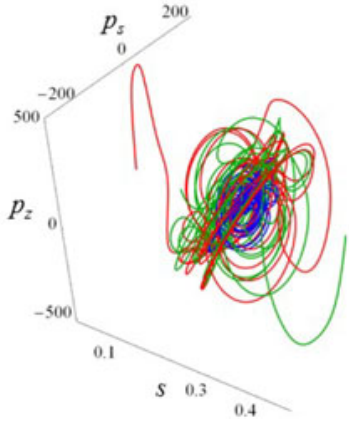

$(f)$

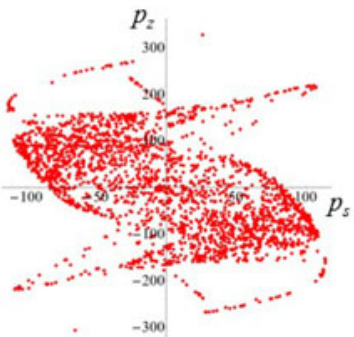

FIGURE 24. Electron trajectories in the case of the second type particular solution corresponding to the motion along the $x=y$ direction in the field of four colliding EM waves for $\varepsilon_{\text {rad }}=1.2 \times 10^{-8}, a_{S}=4 \times 10^{5}, a_{0}=874, \omega=1$ for initial conditions: $x(0)=0.01$, $z(0)=0, p_{x}(0)=0, p_{z}(0)=0($ red, -1$) ; x(0)=0.23, z(0)=0, p_{x}(0)=0, p_{z}(0)=0$ (blue, -2$) ; x(0)=0.45, z(0)=0, p_{x}(0)=0, p_{z}(0)=0$ (green, -3 ). (a) Trajectories in the $(s, z)$ plane. (b) Dependences of the $s$ coordinates on time. (c) Trajectories in the $\left(s, p_{s}, p_{z}\right)$ space. $(d)$ Lorentz factor $\gamma$ versus time for $x(0)=0.45$. (e) Parameter $\chi_{e}$ versus time for $x(0)=0.45$. $(f)$ The Poincare sections: the particle positions in the phase plane $\left(p_{s}, p_{z}\right)$ at discrete times with the time step equal to the period of the driving force for $x(0)=0.01$.

$\left(p_{s}, p_{z}\right)$ at discrete times with the time step equal to the period of the driving force, respectively. According to figure 23(d), where the particle Lorentz factor $\gamma$ is plotted versus time, the normalized electron energy is of the order of the dimensionless EM field amplitude, i.e. $\gamma \approx a_{0}$. From the dependence of the parameter $\chi$ on time in figure 23(e) it follows that, in this case, the QED effect of the recoil is not significant.

The electron interaction with four colliding EM waves in the case of the second type particular solution corresponding to the motion along the $x=y$ direction is illustrated in figure 24 for $\varepsilon_{\text {rad }}=1.2 \times 10^{-8}, a_{S}=4 \times 10^{5}, a_{0}=874, \omega=1$ for initial conditions: $x(0)=0.01, z(0)=0, p_{x}(0)=0, p_{z}(0)=0(\mathrm{red},-1) ; x(0)=0.23$, $z(0)=0, p_{x}(0)=0, p_{z}(0)=0$ (blue, -2$) ; x(0)=0.45, z(0)=0, p_{x}(0)=0, p_{z}(0)=0$ (green, -3). The particle independently of the initial conditions becomes trapped by a strange attractor performing stochastic motion. Panel figure 24(a) shows trajectories in the $(s, z)$ plane. As we see the electrons become trapped in the region of the ponderomotive force minimum. From figure $25(b, c)$ with dependences of the $s$ coordinates on time and with the trajectories in the $\left(s, p_{s}, p_{z}\right)$ space it follows that the trapped particle motion with all the three initial conditions is irregular. As we may see in figure $24(d)$, where the Lorentz factor $\gamma$ versus time for $x(0)=0.23$ is presented, the normalized particle energy is of the order of the dimensionless EM 
(a)

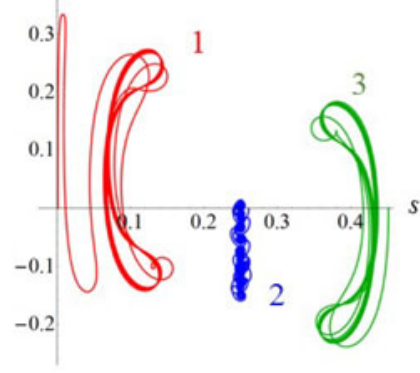

$(d)$

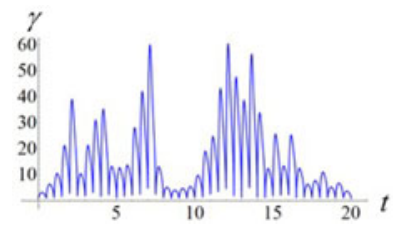

(b)

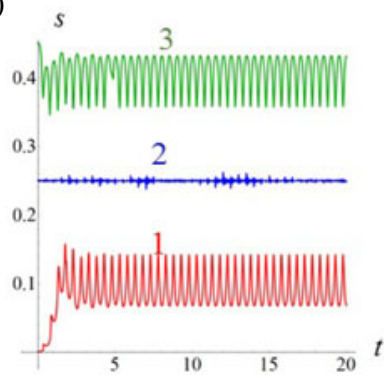

$(e)$

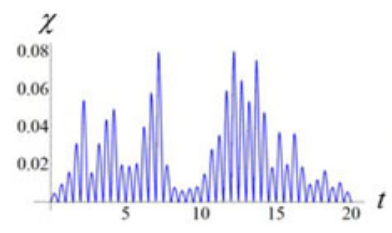

(c)

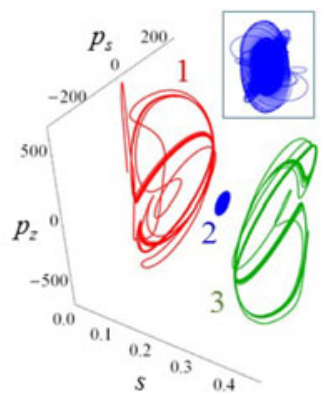

$(f)$

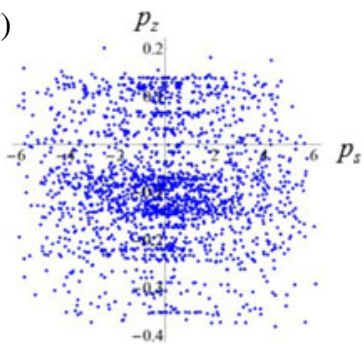

FIGURE 25. Electron trajectories in the case of the second type particular solution corresponding to the motion along the $x=y$ direction in the field of four colliding EM waves for $\varepsilon_{\text {rad }}=3 \times 10^{-9}, a_{S}=1.6 \times 10^{6}, a_{0}=3466, \omega=0.25$ for initial conditions: $x(0)=0.01, z(0)=0, p_{x}(0)=0, p_{z}(0)=0(\mathrm{red},-1) ; x(0)=0.23, z(0)=0, p_{x}(0)=0$, $p_{z}(0)=0$ (blue, -2 ); $x(0)=0.45, z(0)=0, p_{x}(0)=0, p_{z}(0)=0$ (green, -3 ). (a) Trajectories in the $(s, z)$ plane. $(b)$ Dependences of the $s$ coordinates on time. (c) Trajectories in the $\left(s, p_{s}, p_{z}\right)$ space. The inset shows a close up of trajectories in the $\left(s, p_{s}, p_{z}\right)$ for $x(0)=0.23$. $(d)$ Lorentz factor $\gamma$ versus time for $x(0)=0.23$. (e) Parameter $\chi_{e}$ versus time for $x(0)=0.23$. $(f)$ The Poincare sections: the particle positions in the phase plane $\left(p_{s}, p_{z}\right)$ at discrete times with the time step equal to the period of the driving force for $x(0)=0.23$.

wave amplitude. The QED parameter $\chi_{e}$, whose dependence on time for $x(0)=0.23$ is shown in figure 24(e) is lower than unity, i.e. the QED effect of the recoil is weak. The Poincare sections are shown in figure 24(e): the particle positions in the phase plane $\left(p_{s}, p_{z}\right)$ at discrete times with the time step equal to the period of the driving force for $x(0)=0.01$. As we see, the particle motion is stochastic.

Electron interaction with four colliding EM waves in the case of the second type particular solution corresponding to the motion along the $x=y$ direction is illustrated in figure 25 for $\varepsilon_{\text {rad }}=3 \times 10^{-9}, a_{S}=1.6 \times 10^{6}, a_{0}=3466, \omega=0.25$ for initial conditions: $x(0)=0.01, z(0)=0, p_{x}(0)=0, p_{z}(0)=0(\mathrm{red},-1) ; x(0)=0.23, z(0)=0$, $p_{x}(0)=0, p_{z}(0)=0$ (blue, -2$) ; x(0)=0.45, z(0)=0, p_{x}(0)=0, p_{z}(0)=0$ (green, -3$)$. In this case the EM wave frequency is lower than in the above discussed case and the EM wave amplitude is higher. As a result the particle is trapped performing either regular or stochastic motion. Panel figure 25(a) shows trajectories in the $(s, z)$ plane. As we see, depending on the initial conditions, the electron becomes trapped either in the region of the ponderomotive force maximum or in the region of its minimum. From figure $25(b, c)$ with dependences of the $s$ coordinates on time and with the trajectories in the $\left(s, p_{s}, p_{z}\right)$ space it follows that the trapped particle motion 
with the initial conditions $x(0)=0.01$ and $x(0)=0.45$ along the limit circles is regular. As one can see in figure $25(d)$, where the Lorentz factor $\gamma$ versus time for $x(0)=0.23$ is presented, the normalized particle energy is substantially lower than the dimensionless EM wave amplitude. The QED parameter $\chi_{e}$, whose dependence on time for $x(0)=0.23$ is shown figure $25(e)$ is significantly lower than unity, i.e. the QED effect of the recoil is negligibly weak. The Poincare sections are shown in figure 25(e), the particle positions in the phase plane $\left(p_{s}, p_{z}\right)$ at discrete times with the time step equal to the period of the driving force for $x(0)=0.23$. As we see, the particle motion along the trajectories of the attractor plotted in the inset in figure 25(c) with the close up of trajectories in the $\left(s, p_{s}, p_{z}\right)$ for $x(0)=0.23$ is stochastic.

\subsubsection{General case}

The results of integration of the motion equations for the electron interacting with four s-polarized EM waves in the limit of relatively low radiation intensity are presented in figure 26. Figure 26(a) shows 11 electron trajectories in the $(x, y)$ plane for initial conditions as follows. The initial coordinates $x(0)$ and $y(0)$ are chosen to be in the vicinity of the coordinate origin, and $z(0)=0, p_{x}(0)=0, p_{y}(0)=0$, $p_{z}(0)=0$. In figure $26(b)$ we show a close up of the trajectories in the vicinity of the coordinate origin superimposed with the isocontours of the electromagnetic potential averaged over a half-period of the field oscillations. It is proportional to the ponderomotive potential in the high field amplitude limit, $a_{0} \gg 1$. As we see, the typical trajectories are comprised of long range Lévy-flight-like excursions and of short range rambling motion, which changes the direction of the succeeding flight. The corresponding particle trajectory in the $\left(p_{x}, p_{y}, p_{z}\right)$ momentum space for $x(0)=-0.125$ and $y(0)=0.125$ is presented figure $26(c)$. According to the dependence of the parameter $\chi_{e}$ on time plotted in figure 26(d) the QED recoil effects are weak under the conditions of consideration. The Poincare sections, the particle positions in the phase plane $\left(p_{x}, p_{y}\right)$ at discrete times with the time step equal to the period of the driving force, in figure 26(e), show that the particle motion is stochastic.

Figure 27 illustrates the particle dynamics in the EM field formed by four s-polarized EM waves for the radiation intensity higher than that intensity which corresponds to the interaction regime shown in figure 26. Here the electromagnetic field amplitude is $a_{0}=2823$, the dissipation parameter is $\varepsilon_{\text {rad }}=1.2 \times 10^{-9}$, the normalized critical QED field is $a_{S}=4 \times 10^{6}$ and the EM field frequency equals $\omega_{0}=0.1$. From figure $27(a, b)$ it follows that the typical trajectories form a pretty regular pattern in the $(x, y)$ plane. They are comprised of long range Lévy-flight-like excursions and of short range rambling motion, which changes the direction of the succeeding flight. The combination of the long range excursions and short range rambling is also seen in the behaviour of the electron trajectory in the $(x, y, z)$ space presented in figure $27(d)$. The corresponding particle trajectory in the $\left(p_{x}, p_{y}, p_{z}\right)$ momentum space for $x(0)=-0.125$ and $y(0)=0.125$ is presented in figure $27(c)$. What is remarkable is that during the Lévy like flights the electron moves almost along the electric node region, i.e. performing the motion described by the second type particular solution discussed above (see figure 24). The particle normalized energy changes from 200 to approximately 1200 . The value of the QED dimensionless parameter $\chi_{e}$ (not shown here) is less than unity. The Poincare sections (also not shown here) are similar to those sections which are presented in figure 26(e) indicating stochasticity in the electron dynamics. 
(a)

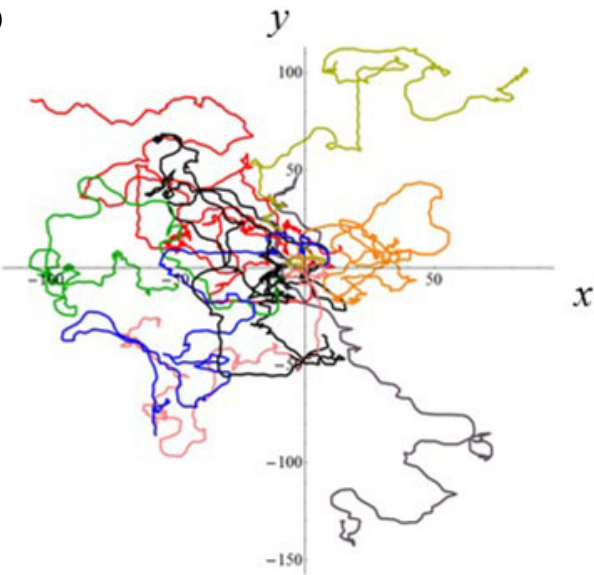

(c)

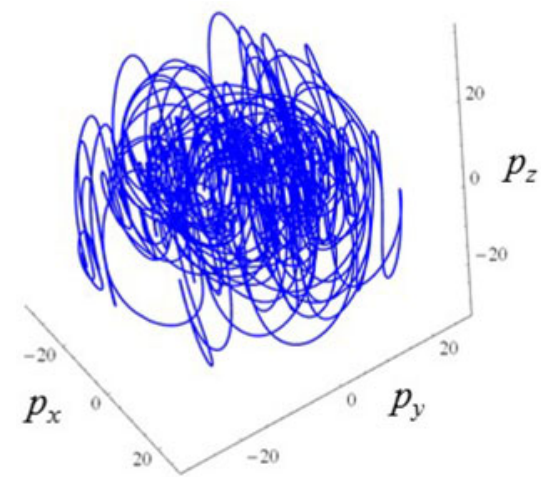

(b)

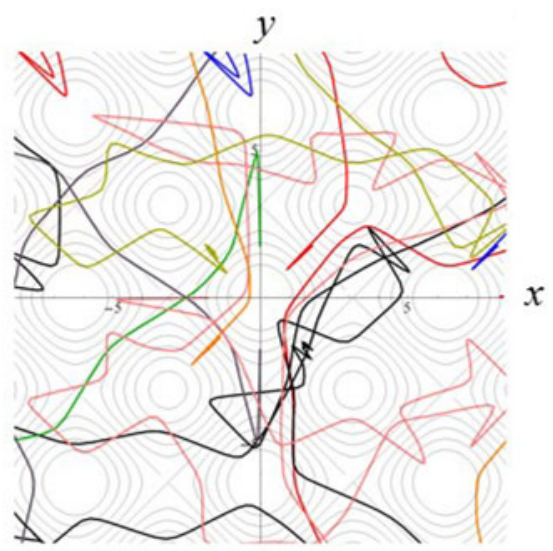

(d)

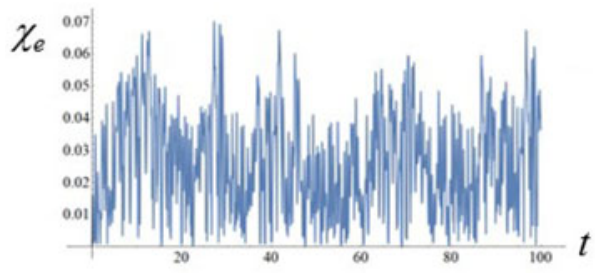

$(e)$

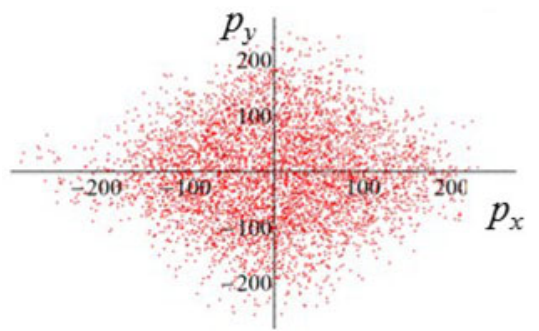

FIgURE 26. (a) Eleven electron trajectories in the $(x, y)$ plane for initial conditions: $x(0)$ and $y(0)$ are in the vicinity of the coordinate origin, and $z(0)=0, p_{x}(0)=0, p_{y}(0)=0$, $p_{z}(0)=0$. (b) Close up of the trajectories in the region $(-7.5<x<7.5 ;-7.5<y<7.5)$. (c) Trajectory in the $\left(p_{x}, p_{y}, p_{z}\right)$ space. $(d)$ Parameter $\chi_{e}$ versus time. $(e)$ The Poincare sections: the particle positions in the phase plane $\left(p_{x}, p_{y}\right)$ at discrete times with the time step equal to the period of the driving force. The electromagnetic field amplitude is $a_{0}=218$, the dissipation parameter is $\varepsilon_{\text {rad }}=1.2 \times 10^{-8}$, the normalized critical QED field is $a_{S}=4 \times 10^{5}$ and the EM field frequency equals $\omega_{0}=1$.

Further increasing the EM field intensity and/or decreasing the field frequency lead to an intriguing change in the trajectory pattern (see figure 28 , where an ensemble of the electron trajectories in the $(x, y)$ plane is presented). The results presented in figures 28 and 29 have been obtained for the electromagnetic field amplitude of $a_{0}=11856$, for the dissipation parameter of $\varepsilon_{\text {rad }}=6 \times 10^{-10}$, for the normalized critical QED field of $a_{S}=8 \times 10^{6}$ and for the EM field frequency equal to $\omega_{0}=0.05$. The trajectory topology can be subdivided into two classes depending on the particle initial conditions. If the particle is initially close to the bottom of the ponderomotive potential, i.e. close to the lines $x= \pm y=\pi n, n=\ldots,-2,-1,0,1,2, \ldots$ in the 
(a)

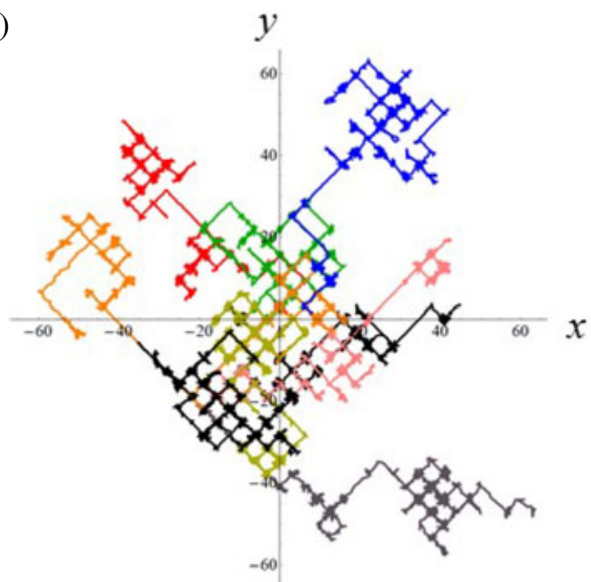

(c)

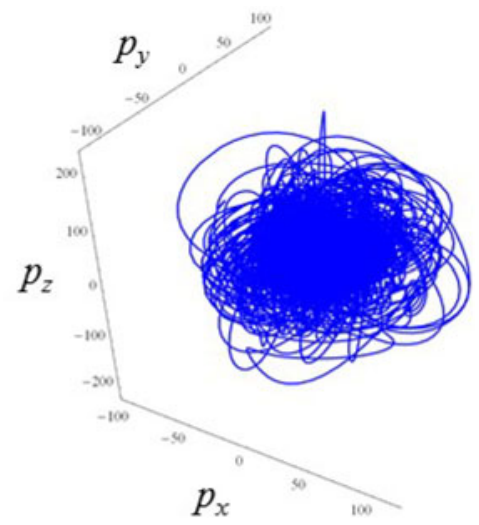

(b)

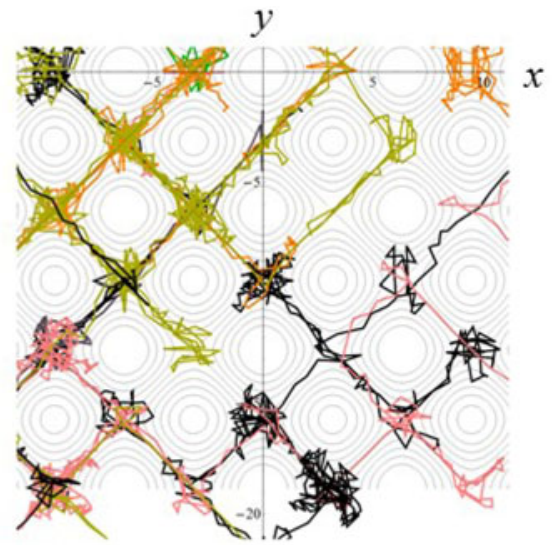

(d)

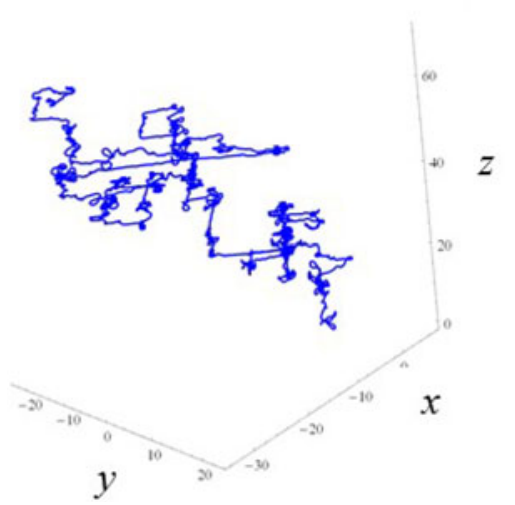

FIGURE 27. (a) Eleven electron trajectories in the $(x, y)$ plane for initial conditions: $x(0)$ and $y(0)$ are in the vicinity of the coordinate origin, and $z(0)=0, p_{x}(0)=0, p_{y}(0)=0$, $p_{z}(0)=0$. (b) Close up of the trajectories in the region $(-10<x<10 ;-20<y<0)$ superimposed with the isocontours if the electromagnetic potential averaged over a halfperiod of the field oscillations. (c) Trajectory in the $\left(p_{x}, p_{y}, p_{z}\right)$ space. $(d)$ Trajectory in the $(x, y, z)$ space. The electromagnetic field amplitude is $a_{0}=2823$, the dissipation parameter is $\varepsilon_{\text {rad }}=1.2 \times 10^{-9}$, the normalized critical QED field is $a_{S}=4 \times 10^{6}$ and the EM field frequency equals $\omega_{0}=0.1$.

$(x, y)$ plane, it remains there. The particle trajectory, in this case, is similar to those shown in figure $27(a, b)$. The second class trajectories are realized for the initial particle positions in the vicinity of the ponderomotive potential maximum, where the magnetic field of the colliding EM waves vanishes. The second class trajectories are trapped within one of the sectors, $0<\theta<\pi / 4, \pi / 4<\theta<\pi / 2$, etc. Oscillating along the radial direction they drift relatively slowly towards the lines either $x=0$ or $y=0$. In both the cases of the first and second topology classes the particles move also along the $z$ axis as seen from the results presented in figure 29. The first class particle dynamics is stochastic: the trajectory in the $\left(p_{x}, p_{y}, p_{z}\right)$ space plotted in figure $29(d)$ corresponds to a strange attractor while figure $29(b)$ shows that the second class dynamics is regular. 


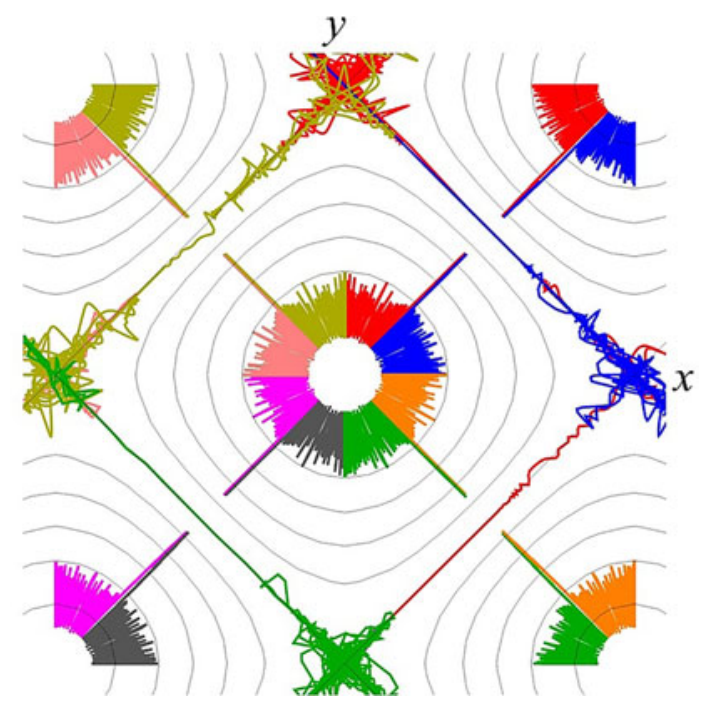

FIGURE 28. Ensemble of the electron trajectories in the $(x, y)$ plane. The particles with the initial coordinates in the region close to the $B=0$ point are trapped inside the sectors, where their trajectories asymptotically approach the lines $x=0$ or $y=0$. For the initial coordinates close to the bottoms of the ponderomotive potential valleys, $x= \pm y=\pi n$, $n=\ldots,-2,-1,0,1,2, \ldots$ the particles move along the trajectories which are similar to those shown in figure $27(a, b)$. The EM field amplitude is $a_{0}=11856$, the dissipation parameter is $\varepsilon_{\text {rad }}=6 \times 10^{-10}$, the normalized critical QED field is $a_{S}=8 \times 10^{6}$ and the EM field frequency equals $\omega_{0}=0.05$.

\subsection{Electron interaction with four p-polarized EM waves}

In the case of four p-polarized colliding laser pulses the EM configuration is described by (6.1)-(6.3). As in the s-polarization case, in the limit of relatively low EM wave intensity the electron performs the random walk motion comprised of short scale-length oscillations interleaved by long scale excursions. An example of such trajectories is shown in figure 30(a) for the EM field amplitude $a_{0}=1.6 \times 10^{3}$, the dissipation parameter equal to $\varepsilon_{\text {rad }}=1.2 \times 10^{-8}$ and the normalized critical QED field of $a_{S}=4.12 \times 10^{5}$. The curve marked by red colour and the number ' 1 ' corresponds to the initial coordinates $x(0)=0.001$ and $y(0)=0.01$. Figure $30(b)$ presents a close up of trajectory $(1)$ the $(x, y)$ plane overlaid with the isocontours of the EM field ponderomotive potential. Electron oscillations in the $\left(p_{x}, p_{y}\right)$ plane (figure $30 c$ ) and dependence of the $y$ coordinate on time plotted in figure $30(d)$ demonstrate that the particle motion is irregular. The stochastic character of the particle dynamics is also distinctly seen in the Poincare sections in the plane $\left(p_{x}, p_{y}\right)$, which is presented in figure $30(d)$.

For ten times higher EM field amplitude, when $a_{0}=1.6 \times 10^{4}$, the particle motion becomes regular as seen in figure 31 . In the $(x, y)$ plane the electron performs long range Lévy-like flights along the lines $x= \pm y+ \pm \pi n$, which end up in the localized attractors, where the particle oscillates pretty regularly (see figure $31 a, b$ ). This electron behaviour is well seen in figure $31(c-e)$ presenting the electron trajectory in the $\left(p_{x}, p_{y}\right)$ plane, the time dependence of the $y$ coordinate and the Poincare mapping in the momentum plane $\left(p_{x}, p_{y}\right)$, respectively. Broadening of the trajectories 
(a)
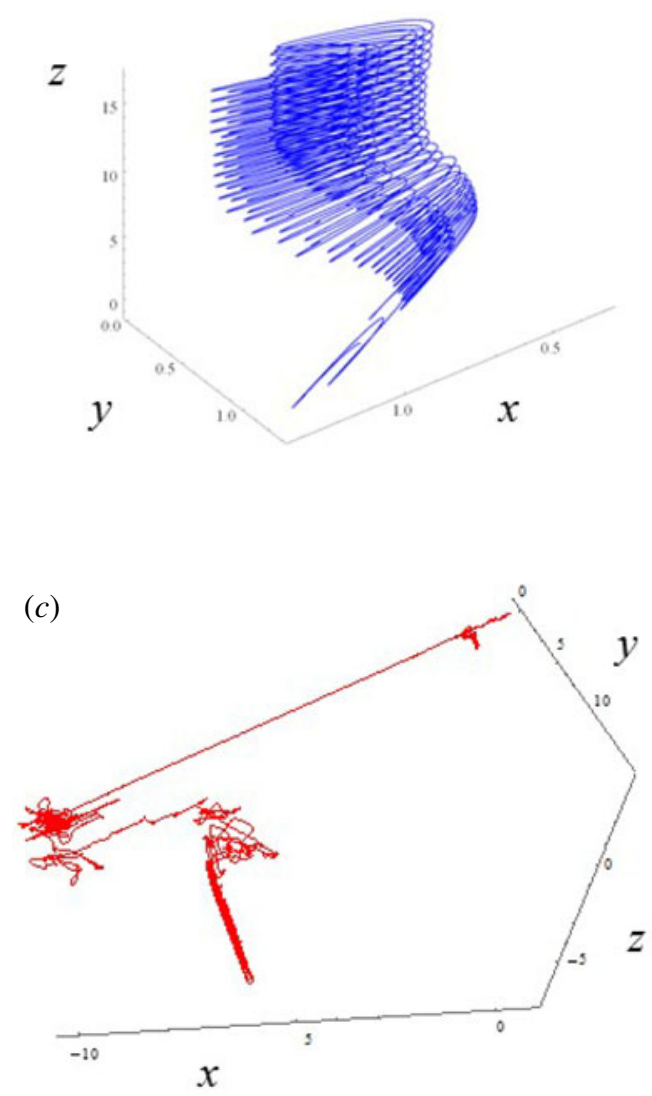

(b)

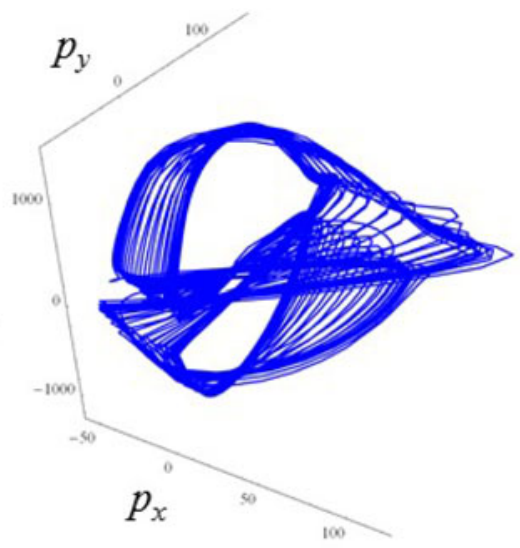

(d)

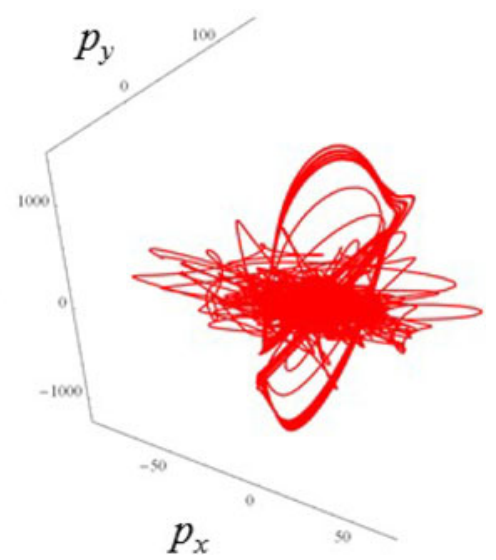

FIGURE 29. (a) Electron trajectory in the $(x, y, z)$ space and $(b)$ trajectory in the $\left(p_{x}, p_{y}, p_{z}\right)$ space for the second class topology. (c) Electron trajectory in the $(x, y, z)$ space and $(d)$ trajectory in the $\left(p_{x}, p_{y}, p_{z}\right)$ space for the first class topology. The electromagnetic field parameters are the same as in figure 28.

in the Poincare mapping figure $31(e)$ also indicates stochastic properties present in the particle motion.

A further ten times increase of the EM field amplitude, $a_{0}=1.6 \times 10^{5}$, results in the particle trapping within narrow stripes localized at the bottoms of the ponderomotive potential (figure $32 a, b$ ). A combination of regular and stochastic aspects of the particle dynamics in this case too is seen from the behaviour of the electron trajectory in the $\left(p_{x}, p_{y}\right)$ plane (figure $\left.32 c\right)$, from the time dependence of the $y$ coordinate (figure $32 d$ ) and from the broadening of the trajectories in the Poincare mapping (figure $32 d$ ).

\section{Conclusions}

As is well known, the multiple colliding laser pulse concept (Bulanov et al. 2010b) is beneficial for achieving an extremely high amplitude of coherent electromagnetic field (see also Bulanov et al. 2010a; Gonoskov et al. 2012, 2013; Gelfer et al. 2015). The complexity of the topology of the time-dependent EM field of colliding laser pulses results in the high complexity of the trajectories of charged particles 
(a) $y$

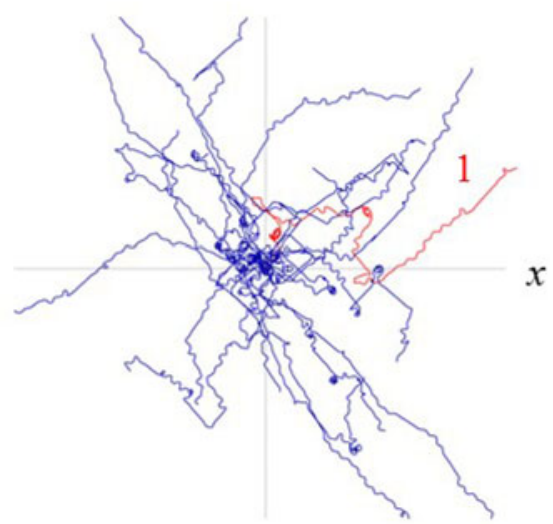

(c)

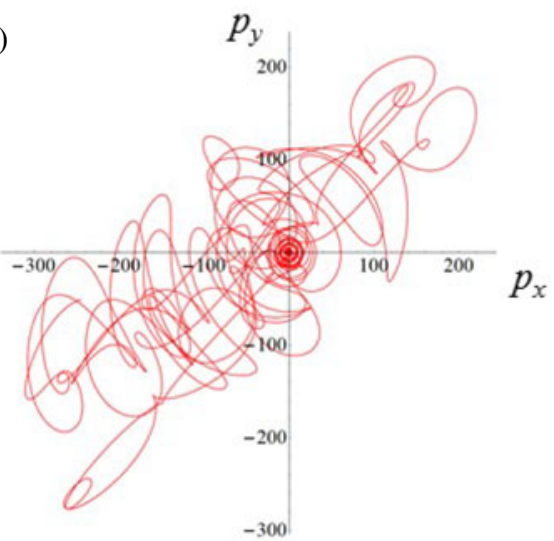

(b)

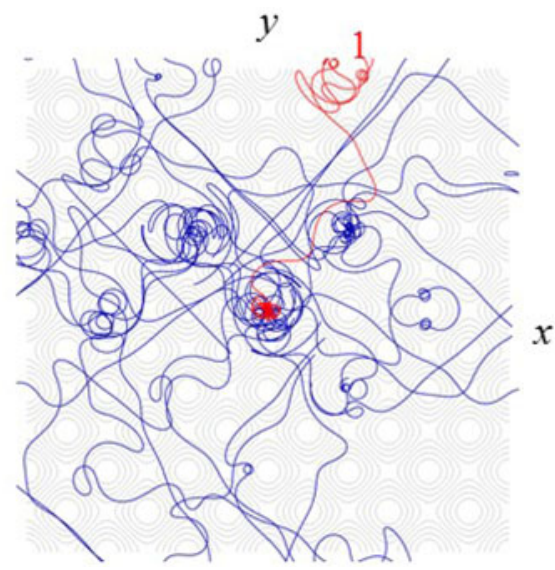

(d)

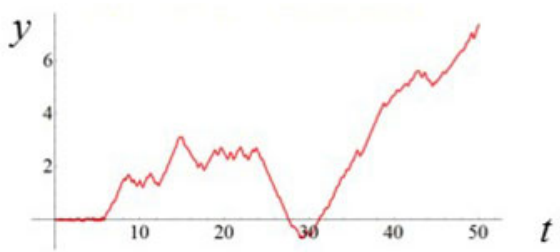

(e)

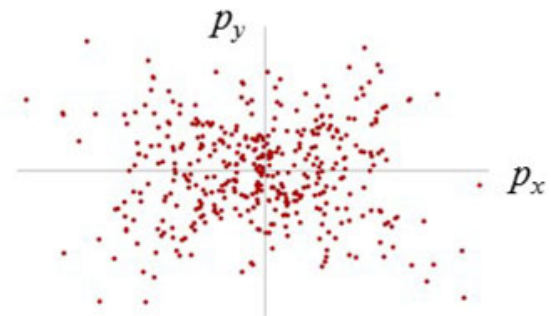

FIGURE 30. Electron interaction with 4 colliding p-polarized EM waves in the low intensity limit for the electromagnetic field amplitude equal to $a_{0}=1.6 \times 10^{3}$, the dissipation parameter equal to $\varepsilon_{\text {rad }}=1.2 \times 10^{-8}$ and the normalized critical QED field of $a_{S}=4.12 \times 10^{5}$. (a) Ensemble of electron trajectories in the $(x, y)$ plane. Red colour (1) curve corresponds to $x(0)=0.001$ and $y(0)=0.01$. (b) Close up of trajectory (1) the $(x, y)$ plane overlaid with the isocontours of the EM field ponderomotive potential. $(c)$ Electron trajectory in the $\left(p_{x}, p_{y}\right)$ plane. $(d)$ Coordinate $y$ versus time $t$. (e) The Poincare sections: the particle positions in the phase plane $\left(p_{x}, p_{y}\right)$ at discrete times with the time step equal to the period of the driving force.

interacting with these fields. In the high field limit, when the radiation friction effects become significant, the charged particle behaviour demonstrates remarkable features corresponding to random walk trajectories, Lévy flights, limit circles, attractors and regular patterns.

In the limit of the relatively weak laser intensity, the electron motion can be described as a random walk figure 5 with the particle over-leaping from one field period to another. The over-leaping correspond to the Lévy flights. In contrast to the standard theory of Lévy flights, which can be found in Lévy (1954), Metzler \& 
(a)

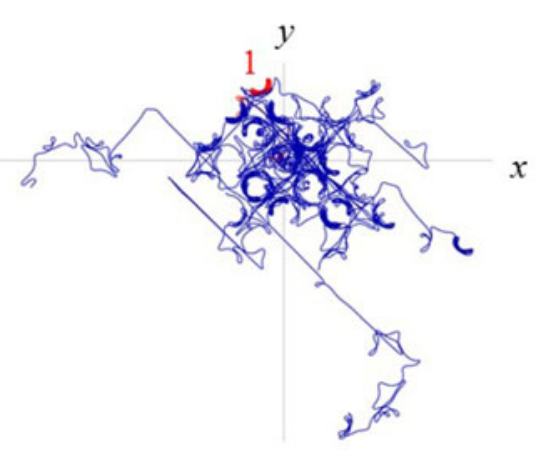

(c)

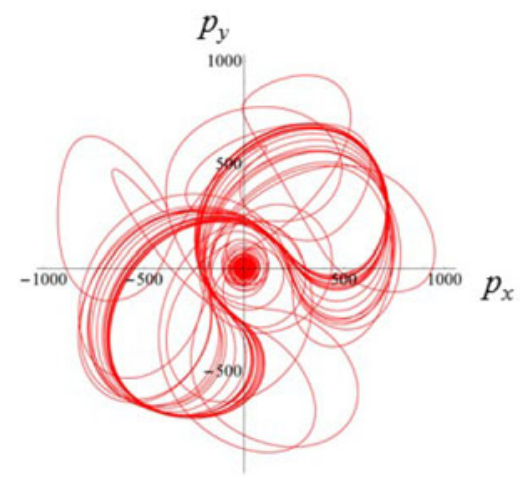

(b)

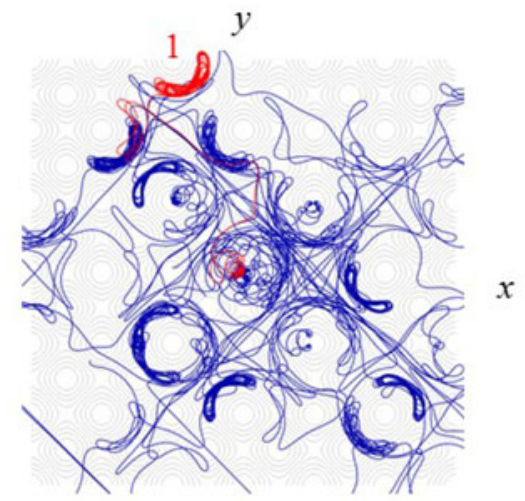

(d)

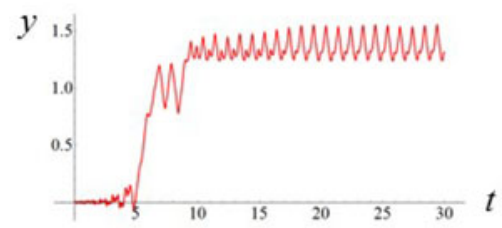

(e)

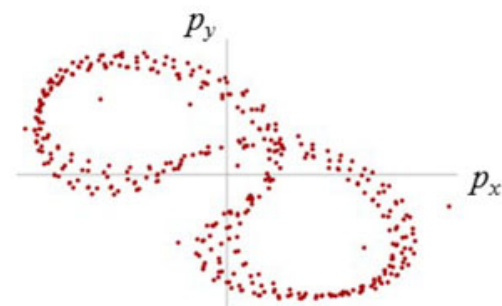

FIgURE 31. The same as in figure 30 for $a_{0}=1.6 \times 10^{4}$.

Klafter (2000), Zaslavsky (2002), Metzler et al. (2007), in the 3 and 4 colliding wave cases considered in the present paper, the Lévy-like flights occur along the directions determined by the landscape of the ponderomotive potential determined in its turn by the geometry of the EM field of the colliding waves. Typically the particle performs short space scale (high frequency) oscillations intermittent with the long range leaps. This oscillation frequency appears to be significantly higher than the frequency of the driver EM wave due to the nonlinearity of the radiation friction force (see also discussion in Esirkepov et al. 2015; Jirka et al. 2016). The length of the long range flight can be found from consideration of the charged particle momentum losses due to radiation friction as in Bulanov et al. (2011a).

Under certain conditions (in the high intensity and/or low frequency limit) the nonlinear dissipation mechanism stabilizes the particle motion causing the particle trapping within a narrow region located near the electric field maximum. In high intensity limit the particle can be trapped in the vicinity of the EM field ponderomotive potential performing regular motion there. The particle trajectory makes regular patterns shown in figures 17 and 28 .

We have analysed the underlying physical mechanism of the radiating charge particle trapping in the regions of the electric field maximum. As elucidated within 
(a)

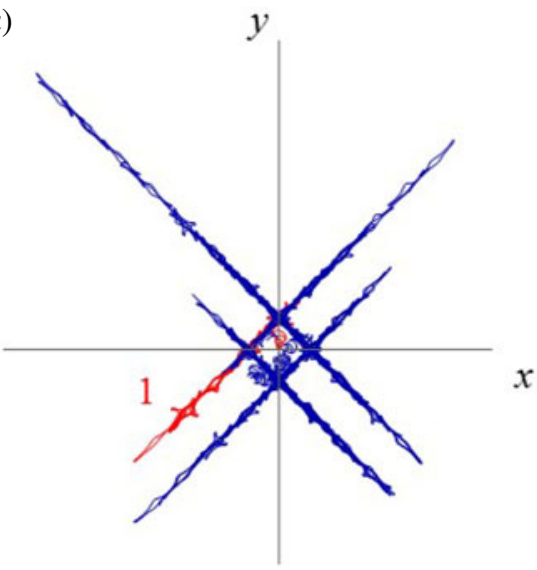

(c)

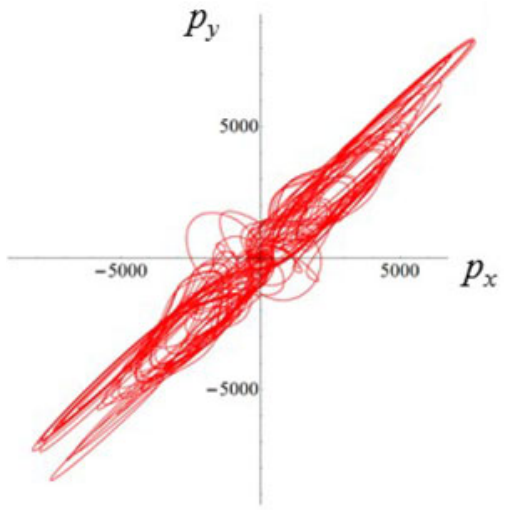

(b)

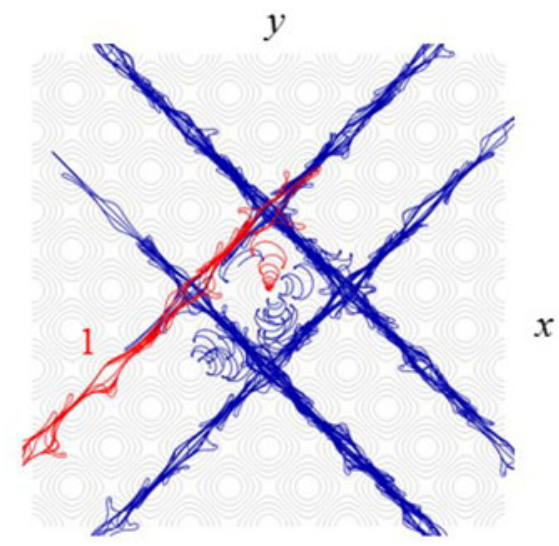

(d)

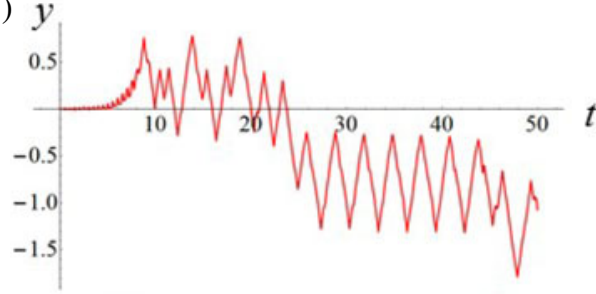

(e)

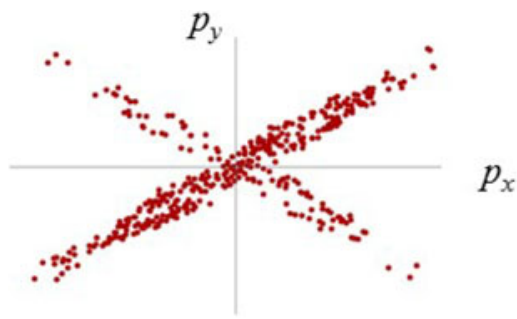

FIGURE 32. The same as in figure 30 for $a_{0}=1.6 \times 10^{5}$.

the framework of the simple model formulated in the present paper the particle trapping is explained by the friction drag originating from the nonlinear dependence of the radiation friction on the EM field.

The attractor trajectory patterns in figures 17, 19 and 27 are made by an ensemble of electrons. The single electron trajectory shown in figure 18 demonstrates that having been moving for a long enough time it could cover the whole attractor. In view of this, there are two questions. The first one being is there an analogy of the ergodic hypothesis saying that over long periods of time, the time spent in some region of the attractor is proportional to the attractor measure? The second one being is there an analogy of the Poincaré recurrence theorem (Arnol'd 1989) saying that the particle, after a sufficiently long but finite time, returns to a point very close to the initial point? A similar question occurs in the case of the particle random walk on whether the results of the well-known random walk theory (see Kac 1961) can be used in our case. Since finding the answers to these questions requires additional thorough consideration, we leave this to the forthcoming publications. 
The structures seen in the electron density distribution obtained in the computer simulations of four electromagnetic pulse interaction with plasma targets (Vranic et al. 2017; Gong et al. 2017) are similar to the structures presented figure 27, which have been found within the framework of a single particle approach. This shows of the robustness of the features discussed above.

One of the most important findings of the present work is a revealing of a new class of regular distributions made by ensembles of the particle trajectories. They are structurally determinate patterns, as if made by tiles, formed in the high field amplitude limit when the radiation friction force drastically modifies the charged particle dynamics in the electromagnetic field as can be distinctly seen in figures 18, 20, 27 and 28. As for the possible practical implications of these findings, these 'crystal-like' patterns are expected to be seen in the spatial distribution of the $\gamma$-rays emitted by the electrons irradiated by the multiple high power laser pulses, which has been noticed in Gong et al. (2017) and Vranic et al. (2017).

\section{Acknowledgements}

S.S.B. acknowledges support from the Office of Science of the US DOE under contract no. DE-AC02-05CH11231. X.Q.Y. and Z.G. acknowledge support from National Basic Research Program of China (grant no. 2013CBA01502), NSFC (grant no. 11535001) and National Grand Instrument Project (2012YQ030142).

\section{REFERENCES}

Abramovitz, M. \& Stegun, I. A. (Eds) 1964 Handbook of Mathematical Functions with Formulas, Graphs, and Mathematical Tables. Dover.

ARNOL'D, V. I. 1989 Mathematical Methods of Classical Mechanics. Springer.

Bashinov, A., Kim, A. \& Sergeev, A. 2015 Impact of quantum effects on relativistic electron motion in a chaotic regime. Phys. Rev. E 92, 043105.

BAshinov, A. V. \& KIM, A. V. 2013 On the electrodynamic model of ultra-relativistic laser-plasma interactions caused by radiation reaction effects. Phys. Plasmas 20, 113111.

Bashinov, A. V., Kumar, P. \& Kim, A. V. 2016 Particle dynamics and spatial e- e+ density structures at qed cascading in circularly polarized standing waves. arXiv:1610.08847.

Bassett, I. M. 1986 Limit to concentration by focusing. Optica Acta: Intl J. Optics 33, 279-286.

Bauer, D., Mulser, P. \& Steeb, W. H. 1995 Relativistic ponderomotive force, uphill acceleration, and transition to chaos. Phys. Rev. Lett. 75, 4622-4625.

Bell, A. R. \& KIRK, J. G. 2008 Possibility of prolific pair production with high-power lasers. Phys. Rev. Lett. 101, 200403.

Beresteskit, V. B., Lifshitz, E. M. \& PitaevskiI, L. P. (Eds) 1982 Quantum Electrodynamics. Pergamon.

Boca, M. \& Florescu, V. 2009 Nonlinear Compton scattering with a laser pulse. Phys. Rev. A 80, 053403.

Bolotovskit, B. M. \& Voskresenski, G. V. 1966 Diffraction radiation. Sov. Phys. Uspekhi 9, 73-96.

Brady, C. S., Ridgers, C. P., Arber, T. D., Bell, A. R. \& KirK, J. G. 2012 Laser absorption in relativistically underdense plasmas by synchrotron radiation. Phys. Rev. Lett. 109, 245006.

Bulanov, S. S., Esirkepov, T., Koga, J., Thomas, A. G. R. \& Bulanov, S. V. $2010 a$ Schwinger limit attainability with extreme power lasers. Phys. Rev. Lett. 105, 220407.

Bulanov, S. S., Mur, V. D., Narozhny, N. B., Nees, J. \& Popov, V. S. $2010 b$ Multiple colliding electromagnetic pulses: a way to lower the threshold of e+ e- pair production from vacuum. Phys. Rev. Lett. 104, 220404. 
Bulanov, S. S., Narozhny, N. B., Mur, V. D. \& Popov, V. S. $2004 a$ On e+ e- pair production by colliding electromagnetic pulses. J. Expl Theor. Phys. Lett. 80, 382-385.

Bulanov, S. S., Narozhny, N. B., Mur, V. D. \& Popov, V. S. 2006 Electron-positron pair production by electromagnetic pulses. J. Expl Theor. Phys. 102, 9-23.

Bulanov, S. S., Schroeder, C. B., Esarey, E. \& Leemans, W. P. 2013 Electromagnetic cascade in high-energy electron, positron, and photon interactions with intense laser pulses. Phys. Rev. A 87, 062110.

Bulanov, S. V. 2017 Magnetic reconnection: from MHD to QED. Plasma Phys. Control. Fusion 59, 014029.

Bulanov, S. V., Esirkepov, T. Zh., Hayashi, Y., Kando, M., Kiriyama, H., Koga, J. K., Kondo, K., Kotaki, H., Pirozhkov, A. S., Bulanov, S. S. et al. 2011 a On the design of experiments for the study of extreme field limits in the interaction of laser with ultrarelativistic electron beam. Nucl. Instrum. Meth. Phys. Res. A 660, 31-42.

Bulanov, S. V., Esirkepov, T. Zh., Kando, M., Koga, J., Kondo, K. \& Korn, G. 2015 On the problems of relativistic laboratory astrophysics and fundamental physics with super powerful lasers. Plasma Phys. Rep. 41, 1-51.

Bulanov, S. V., Esirkepov, T. Zh., Kando, M., Koga, J. K. \& Bulanov, S. S. $2011 b$ Lorentz-Abraham-Dirac versus Landau-Lifshitz radiation friction force in the ultrarelativistic electron interaction with electromagnetic wave (exact solutions). Phys. Rev. E 84, 056605.

Bulanov, S. V., Esirkepov, T. Zh., Koga, J. \& Tajima, T. $2004 b$ Interaction of electromagnetic waves with plasma in the radiation-dominated regime. Plasma Phys. Rep. 30, 196-213.

Chang, H. X., QiaO, B., Xu, Z., Xu, X. R., Zhou, C. T., Yan, X. Q., Wu, S. Z., Borghesi, M., ZEPF, M. \& HE, X. T. 2015 Generation of overdense and high-energy electron-positron-pair plasmas by irradiation of a thin foil with two ultraintense lasers. Phys. Rev. E 92, 053107.

Corvan, D. J., ZEPF, M. \& SARRI, G. 2016 A high-energy, high-flux source of gamma-rays from all-optical non-linear Thomson scattering. Nucl. Instrum. Meth. Phys. Res. A 829, 291-300.

Di PiazzA, A. 2008 Exact solution of the Landau-Lifshitz equation in a plane wave. Lett. Math. Phys. 83, 305-315.

Di Piazza, A., Hatsagortsyan, K. Z. \& Keitel, C. H. 2010 Quantum radiation reaction effects in multiphoton compton scattering. Phys. Rev. Lett. 105, 220403.

Di Piazza, A., Muller, C., Hatsagortsyan, K. Z. \& Keitel, C. H. 2012 Extremely highintensity laser interactions with fundamental quantum systems. Rev. Mod. Phys. 84, 1177-1228.

Duclous, R., KirK, J. G. \& Bell, A. R. 2011 Monte Carlo calculations of pair production in high-intensity laser-plasma interactions. Plasma Phys. Control. Fusion 53, 015009.

Durrett, R. 1991 Probability. Theory and Examples. Wadsworth \& Brooks/Cole.

Ehlotzky, F., KRAJeWska, K. \& KAMIŚKi, J. Z. 2009 Fundamental processes of quantum electrodynamics in laser fields of relativistic power. Rep. Prog. Phys. 72, 046401.

Elkina, N. V., Fedotov, A. M., Kostyukov, I. Yu., Legkov, M. V., Narozhny, N. B., Nerush, E. N. \& RUhL, H. 2011 QED cascades induced by circularly polarized laser fields. Phys. Rev. STAB 14, 054401.

ERBER, T. 1966 High-energy electromagnetic conversion processes in intense magnetic fields. Rev. Mod. Phys. 38, 626-659.

Esirkepov, T. Zh., Bulanov, S. S., Koga, J., Kando, M., Kondo, K., Rosanov, N. N., Korn, G. \& Bulanov, S. V. 2015 Attractors and chaos of electron dynamics in electromagnetic standing waves. Phys. Lett. A 379, 2044-2054.

Esirkepov, T. ZH. \& Bulanov, S. V. 2017 Paradoxical stabilization of forced oscillations by strong nonlinear friction. arXiv:1702.01645.

Fedotov, A. M., Elkina, N. V., Gelfer, E. G., Narozhny, N. B. \& Ruhl, H. 2014 Radiation friction versus ponderomotive effect. Phys. Rev. A 90, 053847.

Fedotov, A. M., Narozhny, N. B., Mourou, G. \& Korn, G. 2010 Limitations on the attainable intensity of high power lasers. Phys. Rev. Lett. 105, 080402.

Gales, S., Balabanski, D. L., Negoita, F., Tesileanu, O., Ur, C. A., Ursescu, D. \& ZAMFIR, N. V. 2016 New frontiers in nuclear physics with high-power lasers and brilliant monochromatic gamma beams. Phys. Scr. 91, 093004. 
Gelfer, E. G., Mironov, A. A., Fedotov, A. M., Bashmakov, V. F., Nerush, E. N., Kostyukov, I. Yu. \& NArozhny, N. B. 2015 Optimized multibeam configuration for observation of qed cascades. Phys. Rev. A 92, 022113.

Gong, Z., Hu, R., Shou, Y., Qiao, B., Chen, C., Xu, F., He, X. \& Yan, X. 2016 Radiation reaction induced spiral attractors in ultra-intense colliding laser beams. Matt. Radiat. Extremes 1, 308-315.

Gong, Z., Hu, R. H., Shou, Y. R., Qiao, B., Chen, C. E., He, X. T., Bulanov, S. S., ESIRKEPOV, T. ZH., BUlANOV, S. V. \& YAN, X. Q. 2017 High efficiency gamma-ray flash generation via multiple Compton scattering in ponderomotive potential well. Phys. Rev. E 95, 013210.

Gonoskov, A., Bashinov, A., Bastrakov, S., Efimenko, E., Ilderton, A., Kim, A., Marklund, M., Meyerov, I., Muraviev, A. \& Sergeev, A. 2016 Ultra-bright GeV photon source via controlled electromagnetic cascades in laser-dipole waves. arXiv:1610.06404v1 [physics.plasm-ph].

Gonoskov, A., Bashinov, A., Gonoskov, I., Harvey, C., Ilderton, A., Kim, A., Marklund, M., Mourou, G. \& SergeEv, A. 2014 Anomalous radiative trapping in laser fields of extreme intensity. Phys. Rev. Lett. 113, 014801.

Gonoskov, A., Gonoskov, I., Harvey, C., Ilderton, A., Kim, A., Marklund, M., Mourou, G. \& SERgeEv, A. 2013 Probing nonperturbative qed with optimally focused laser pulses. Phys. Rev. Lett. 111, 060404.

Gonoskov, I., Aiello, A., Heugel, S. \& Leuchs, G. 2012 Dipole pulse theory: maximizing the field amplitude from $4 \mathrm{p}$ focused laser pulses. Phys. Rev. A 86, 053836.

Grismayer, T., Vranic, M., Martins, J. L., Fonseca, R. A. \& Silva, L. O. 2016 Laser absorption via quantum electrodynamics cascades in counter propagating laser pulses. Phys. Plasmas 23, 056706.

Harvey, C., Heinzl, T. \& Ilderton, A. 2009 Signatures of high-intensity Compton scattering. Phys. Rev. A 79, 063407.

Harvey, C., Heinzl, T. \& Marklund, M. 2011 Symmetry breaking from radiation reaction in ultra-intense laser fields. Phys. Rev. D 84, 116005.

Heinzl, T., Harvey, C., Ilderton, A., Marklund, M., Bulanov, S. S., Rykovanov, S., Schroeder, C. B., Esarey, E. \& Leemans, W. P. 2015 Detecting radiation reaction at moderate laser intensities. Phys. Rev. E 91, 023207.

Heinzl, T., Ilderton, A. \& MARKLund, M. $2010 a$ Finite size effects in stimulated laser pair production. Phys. Lett. B 692, 250-256.

Heinzl, T., SEIPT, D. \& KÄMPfer, B. $2010 b$ Beam-shape effects in nonlinear Compton and Thomson scattering. Phys. Rev. A 81, 022125.

ILderton, A. \& Torgrimsson, G. 2013 Scattering in plane-wave backgrounds: infrared effects and pole structure. Phys. Rev. D 88, 025021.

Ji, L. L., Pukhov, A., Kostyukov, I. Yu., Shen, B. F. \& Akli, K. 2014 Radiation-reaction trapping of electrons in extreme laser fields. Phys. Rev. Lett. 112, 145003.

Jirka, M., Klimo, O., Bulanov, S. V., Esirkepov, T. Zh., Gelfer, E., Bulanov, S. S., Weber, S. \& Korn, G. 2016 Electron dynamics and gamma- and e- e+ production by colliding laser pulses. Phys. Rev. E 93, 023207.

KAC, M. 1961 Kilka zagadnien stochastycznych fizyki matematyki. Panstw. Wydaw. Nauk.

KIRK, J. G. 2016 Radiative trapping in intense laser beams. Plasma Phys. Control. Fusion 58, 085005 .

KogA, J. K. 2004 Integration of the Lorentz-Dirac equation: interaction of an intense laser pulse with high-energy electrons. Phys. Rev. E 70, 046502.

Koga, J. K., Esirkepov, T. Zh. \& Bulanov, S. V. 2005 Nonlinear Thomson scattering in the strong radiation damping regime. Phys. Plasmas 12, 093106.

Koga, J. K., Esirkepov, T. ZH. \& Bulanov, S. V. 2006 Nonlinear Thomson scattering with strong radiation damping. J. Plasma Phys. 72, 1315-1318.

Krajewska, K. \& Kamiński, J. Z. 2012 Breit-Wheeler process in intense short laser pulses. Phys. Rev. A 85, 062102. 
Landau, L. D. \& Lifshitz, E. M. 1976 Mechanics. Pergamon.

Landau, L. D. \& Lifshitz, E. M. (Eds) 1982 The Classical Theory of Field. Pergamon.

Lehmann, G. \& SpatscheK, K. H. 2012 Phase-space contraction and attractors for ultrarelativistic electrons. Phys. Rev. E 85, 056412.

Lehmann, G. \& SpatscheK, K. H. 2016 Transient plasma photonic crystals for high-power lasers. Phys. Rev. Lett. 116, 225002.

Levy, M. C., Blackburn, T. G., Ratan, N., Sadler, J., Ridgers, C. P., Kasim, M., Ceurvorst, L., Holloway, J., Baring, M. G., Bell, A. R. et al. 2016 QED-driven laser absorption. arXiv:1609.00389v1 [physics.plasm-ph].

LÉVY, P. P. 1954 Théorie de l'addition des variables aléatoires, 2nd edn. Gauthier-Villars.

Liseykina, T. V., Popruzhenko, S. V. \& MACChi, A. 2016 Inverse Faraday effect driven by radiation friction. New J. Phys. 18, 072001.

Lobet, M., Ruyer, C., Debayle, A., D’Humiéres, E., Grech, M., Lemoine, L. \& Gremillet, L. 2015 Ultrafast synchrotron-enhanced thermalization of laser-driven colliding pair plasmas. Phys. Rev. Lett. 115, 215003.

Mackenroth, F. \& Di PiazZA, A. 2011 Nonlinear Compton scattering in ultrashort laser pulses. Phys. Rev. A 83, 032106.

Marklund, M. \& Shukla, P. 2006 Nonlinear collective effects in photon-photon and photon-plasma interactions. Rev. Mod. Phys. 78, 591-640.

Mathur, M., Haller, G., Peacock, T., Ruppert-Felsot, J. E. \& Swinney, H. L. 2007 Uncovering the Lagrangian skeleton of turbulence. Phys. Rev. Lett. 98, 144502.

MendoncA, J. T. 1983 Threshold for electron heating by two electromagnetic waves. Phys. Rev. A 28, 3592-3598.

Metzler, R., Chechin, A. V., Gonchar, V. Yu. \& Klafter, J. 2007 Some fundamental aspects of Lévy flights. Chaos Solitons Fractals 34, 129-142.

Metzler, R. \& Klafter, J. 2000 The random walk's guide to anomalous diffusion: a fractional dynamics approach. Phys. Rep. 339, 1-77.

Mourou, G., Tajima, T. \& Bulanov, S. V. 2006 Optics in the relativistic regime. Rev. Mod. Phys. 78, 309-371.

Mourou, G. A., Korn, G., SAndner, W. \& Collier, J. L. (Eds) 2011 ElI-Extreme Light Infrastructure Science and Technology with Ultra-Intense Lasers WHITEBOOK. THOSS Media $\mathrm{GmbH}$.

Nakamura, T., Koga, J. K., Esirkepov, T. Z., Kando, M., Korn, G. \& Bulanov, S. V. 2012 High-power gamma-ray flash generation in ultraintense laser-plasma interactions. Phys. Rev. Lett. 108, 195001.

Narozhnyi, N. B. \& Fofanov, M. S. 1996 Photon emission by an electron in a collision with a short focused laser pulse. J. Expl Theor. Phys. 83, 14-23.

Nerush, E. N., Kostyukov, I. Yu., Fedotov, A. M., Narozhny, N. B., Elkina, N. V. \& RuHL, H. 2011 Laser field absorption in self-generated electron-positron pair plasma. Phys. Rev. Lett. 106, 035001.

Nikishov, A. I. \& Ritus, V. I. $1964 a$ Quantum processes in the field of a plane electromagnetic wave and in a constant field. I. Sov. Phys. JETP 19, 529-541.

Nikishov, A. I. \& Ritus, V. I. $1964 b$ Quantum processes in the field of a plane electromagnetic wave and in a constant field. II. Sov. Phys. JETP 19, 1191-1199.

Peacock, T. \& Haller, G. 2013 The hidden skeleton of fluid flows. Phys. Today 66, 41-47.

Ridgers, C. P., Brady, C. S., Duclous, R., Kirk, J. G., Bennett, K., Arber, T. D., Robinson, A. \& Bell, A. 2012 Dense electron-positron plasmas and ultraintense gamma rays from laser-irradiated solids. Phys. Rev. Lett. 108, 165006.

Ritus, V. I. 1985 Quantum effects of the interaction of elementary particles with an intense electromagnetic field. J. Sov. Laser Res. 6, 497-617.

SCHWINGER, J. 1954 The quantum correction in the radiation by energetic accelerated electrons. Proc. Natl Acad. Sci. USA 40, 132-136.

Sheng, Z.-M., Mima, K., Sentoku, Y., Jovanovic, M. S., Taguchi, T., Zhang, J. \& MeyerTER VEHN, J. 2002 Stochastic heating and acceleration of electrons in colliding laser fields in plasma. Phys. Rev. Lett. 88, 055004. 
Sokolov, A. A., Klepikov, N. P. \& Ternov, I. M. 1952 Zur quantentheorie des energieverlustes von elektronen im magnetfeld. Zh. Ehksp. Teor. Fiz. 23, 632-641.

Sokolov, I. V., Nees, J. A., Yanovsky, V. P., Naumova, N. M. \& Mourou, G. A. 2010 Emission and its back-reaction accompanying electron motion in relativistically strong and qed-strong pulsed laser fields. Phys. Rev. E 81, 036412.

Tamburini, M., Pegoraro, F., Di Piazza, A., Keitel, C. H., Liseykina, T. V. \& Macchi, A. 2011 Radiation reaction effects on electron nonlinear dynamics and ion acceleration in laser-solid interaction. Nucl. Instrum. Meth. Phys. Res. A 653, 181.

Tamburini, M., Pegoraro, F., Di Piazza, A., Keitel, C. H. \& Macchi, A. 2010 Radiation reaction effects on radiation pressure acceleration. New. J. Phys. 12, 123005.

TAng, S., Kumar, N. \& Keitel, C. H. 2016 Optimized plasma high harmonics generation from ultra-intense laser pulses. arXiv:1608.02513v1 [physics.plasm-ph].

Thomas, A. G. R., Ridgers, C. P., Bulanov, S. S., Griffin, B. J. \& Mangles, S. P. D. 2012 Strong radiation-damping effects in a gamma-ray source generated by the interaction of a high-intensity laser with a wakefield-accelerated electron beam. Phys. Rev. X 2, 041004.

Thomson, W. (LoRd Kelvin) 1884 Notes of Lectures on Molecular Dynamics and the Wave Theory of Light. Delivered at The Johns Hopkins University, Baltimore, by Sir William Thomson, Professor in the University of Glasgow. Stenographically Reported by A. S. Hathaway, Lately Fellow in Mathematics of The Johns Hopkins University. Johns Hopkins.

Titov, A. I., TAKabe, H., Kämpfer, B. \& HosaKa, H. 2012 Enhanced subthreshold e+ eproduction in short laser pulses. Phys. Rev. Lett. 108, 240406.

Vranic, M., Grismayer, T., Fonseca, R. A. \& Silva, L. O. 2017 Electron-positron cascades in multiple-laser optical traps. Plasma Phys. Control. Fusion 59, 014040.

Zaslavsky, G. M. 2002 Chaos, fractional kinetics, and anomalous transport. Phys. Rep. 371, 461-580.

ZeL'Dovich, YA. B. 1975 Interaction of free electrons with electromagnetic radiation. Sov. Phys. Uspekhi 18, 79-98.

Zhang, S. 2013 Pre-acceleration from Landau-Lifshitz series. Prog. Theor. Exper. Physics 123, $123 \mathrm{~A} 01$.

Zhidkov, A. G., Koga, J., SASAKi, A. \& UesakA, M. 2002 Radiation damping effects on the interaction of ultraintense laser pulses with an overdense plasma. Phys. Rev. Lett. 88, 185002. 